\title{
STRATEGICALLY REPRODUCIBLE BASES AND THE FACTORIZATION PROPERTY
}

\author{
R. LECHNER, P. MOTAKIS, P.F.X. MÜLLER, AND TH. SCHLUMPRECHT
}

\begin{abstract}
We introduce the concept of strategically reproducible bases in Banach spaces and show that operators which have large diagonal with respect to strategically reproducible bases are factors of the identity. We give several examples of classical Banach spaces in which the Haar system is strategically reproducible: multi-parameter Lebesgue spaces, mixed-norm Hardy spaces and most significantly the space $L^{1}$. Moreover, we show the strategical reproducibility is inherited by unconditional sums.
\end{abstract}

\section{INTRODUCTION}

In this paper, we address the following question: Given a Banach space $X$ with a basis $\left(e_{i}\right)_{i=1}^{\infty}$, let $T: X \rightarrow X$ be an operator, whose matrix representation has a diagonal whose elements are uniformly bounded away from 0 . We say in that case $T$ has a large diagonal. Is it possible to factor the identity operator on $X$ through $T$ ?

The origin of this problem can be traced back to the work of Pełczyński 24], who proved that every infinite dimensional subspace of $\ell^{p}, 1 \leq p<\infty$ and $c_{0}$ contains a further subspace which is complemented and isomorphic to the whole space.

Closely related is the concept of primarity of a Banach space. Recall that $X$ is called primary, if for every bounded projection $P: X \rightarrow X$, either $P(X)$ or $(I-P)(X)$ is isomorphic to $X$. The connection between the primarity of a Banach space and the factorization problem is as follows: either $P$ has large diagonal or $I-P$ has large diagonal on a "large" subsequence of the basis $\left(e_{i}\right)_{i=1}^{\infty}$ of the Banach space $X$. For example Maurey [17 proved primarity for $X=L^{p}, 1 \leq p<\infty$, by showing that for every operator $T: L^{p} \rightarrow L^{p}$, the identity operator factors either through $T$ or $I-T$; see also Alspach-Enflo-Odell 22. Factorization and primarity theorems were obtained by Capon [4] for the mixed norm spaces $L^{p}\left(L^{q}\right), 1<p, q<\infty$, and by the third named author [19] for $H^{1}$ and BMO.

Separately, Andrew [1] showed that for $1<p<\infty$, every operator $T: L^{p} \rightarrow L^{p}$ which has large diagonal with respect to the Haar system is a factor of the identity operator on $L^{p}$. More recently in 13 it was proved that for $1 \leq p, q<\infty$, every operator $T: H^{p}\left(H^{q}\right) \rightarrow H^{p}\left(H^{q}\right)$ which has large diagonal is a factor of the identity operator on $H^{p}\left(H^{q}\right)$.

In this paper we introduce a new approach to the factorization problem by devising an infinite two person game and isolate a property of a basis called strategical

2010 Mathematics Subject Classification. 46B25, 47A68, 30H10.

The first and the third author was supported by the Austrian Science Foundation (FWF) under Grant Number Pr.Nr. P28352. The second named author was supported by the National Science Foundation under Grant Number DMS-1600600. The fourth named author was supported by the National Science Foundation under Grant Numbers DMS-1464713 and DMS-1711076 . 
reproducibility, which implies the factorization through the identity of operators with large diagonal. We say in that case, the basis $\left(e_{i}\right)_{i=1}^{\infty}$ has the factorization property. By using this method, we obtain simplified proofs of existing results, and obtain the following new factorization theorems for $L^{1}$ and related spaces.

Theorem. The normalized Haar system of $L^{1}[0,1]$ has the factorization property. Moreover, the normalized bi-parameter Haar system of $L^{1}\left([0,1]^{2}\right)$ and the tensor product of the $\ell^{p}$ unit vector basis with the Haar system have the factorization property.

The paper is organized as follows. Section 2 covers basic concepts relevant to this work. In Section 3, we define three notions of strategical reproducibility and show that those imply the factorization property. In Section 4 we review basic properties of multi-parameter Lebesgue- and Hardy spaces. In Section 5 we establish that the Haar system is strategically reproducible in several classical Banach spaces such as reflexive, multi-parameter Lebesgue spaces, $H^{1}$ and two-parameter Hardy spaces $H^{p}\left(H^{q}\right), 1 \leq p, q<\infty$. In Section 6 we show that the Haar system is strategically reproducible in $L_{0}^{1}$. In Section 7 we show that unconditional sums of spaces with strategically reproducible bases have themselves that property. Finally, we discuss open problems in Section 8 .

\section{A BRIEF DISCUSSION OF BASIC CONCEPTS}

We discuss several closely related concepts for operators on Banach spaces.

Definition 2.1. Let $X$ be a Banach space and $T: X \rightarrow X$ be a bounded linear operator.

(i) We say that $T(X)$ contains a copy of $X$ if there is a (necessarily closed) subspace $Y$ of $T(X)$ that is isomorphic to $X$.

(ii) We say that $T$ preserves a copy of $X$ (or fixes a copy of $X$ ) if there exists a subspace $Y$ of $X$ that is isomorphic to $X$ and $T$ restricted on $Y$ is an into isomorphism.

(iii) We say that the identity operator $I$ on $X$ factors through $T$ if there are bounded linear operators $R, S: X \rightarrow X$ with $I=S T R$.

We also consider a quantified version of (iii). For $K>0$ we say that the identity $K$-factors through $T$ if there are bounded linear operators $R, S: X \rightarrow X$ with $\|R\| \cdot\|S\| \leq K$ and $I=S T R$ and we say that the identity almost $K$-factors through $T$ if it $(K+\varepsilon)$-factors through $T$ for all $\varepsilon>0$.

Remark 2.2. In general, for a given operator $T$, it is easy to see that (iii) $\Rightarrow$ (ii) and (ii) $\Rightarrow$ (i). The converse implications are in general false. To see that (i) $\nRightarrow$ (ii) take a quotient operator $T_{0}: L^{1} \rightarrow \ell_{1}$ and a quotient operator $T_{1}: \ell_{1} \rightarrow L^{1}$. Then if $T=T_{1} \circ T_{0}: L^{1} \rightarrow L^{1}, T\left(L^{1}\right)=L^{1}$ however $T$ does not preserve a copy of $L^{1}$. There is an example demonstrating (iii) $\nRightarrow$ (ii) but it is slightly more involved. We first observe that if $I=S T R$ and $Z=T R(X)$, then $Z$ is isomorphic to $X$ and complemented in $X$. Indeed, it follows that $R$ is bounded below and $T$ is bounded below on $R(X)$ hence $T R$ is an isomorphic embedding. Furthermore, $S$ restricted on $Z=T R(X)$ is an isomorphism onto $X$. Therefore, we can define the inverse map $\left.S\right|_{Z} ^{-1}: X \rightarrow Z$. One can check that $P x=\left.S\right|_{Z} ^{-1}(S x)$ defines a bounded projection onto $Z$. This easy fact implies that if $X$ is a minimal space that is not complementably minimal then there exists an operator $T: X \rightarrow X$ that is an into 
isomorphism so that the identity does not factor through $X$. To see this, choose a subspace $Y$ of $X$ that is isomorphic to $X$ and does not contain a further subspace isomorphic to $X$ and complemented in $X$. If $T: X \rightarrow X$ is an into isomorphism, the image of which is $Y$, then the identity does not factor through $T$. Indeed, if $I=S T R$ then $Z=T R(X)$ is isomorphic to $X$ and complemented to $X$. This is not possible because $Z$ is a subspace of $Y$. In conclusion, the fact that (iii) $\nRightarrow$ (ii) is reduced to the existence of a minimal and not complementably minimal space $X$. It is well known that the dual of Tsirelson space has this property, however to the best of our knowledge there is no recorded proof of this fact so we give a short description of it here. Assume that $T^{*}$ is complementably minimal. We will show that this would imply that $T$ is minimal, which was proved to be false in 7. Corollary VI.b.6, page 58]. Let $X$ be an infinite dimensional subspace of $T$. By [6, Theorem 1] $X$ is isomorphic to a quotient of $T$ and hence $X^{*}$ is isomorphic to a subspace of $T^{*}$. If $T^{*}$ is complementably minimal, then $X^{*}$ contains a complemented copy of $T^{*}$ which yields that $X$ contains a complemented copy of $T$. In particular, $T$ is minimal and this cannot be the case.

The following definition of $C$-perturbable was introduced by Andrew in 1. The concept of large diagonal, which was implicitly present in [1], was formally introduced in 13 .

Definition 2.3. Let $X$ be a Banach space with a normalized Schauder basis $\left(e_{k}\right)_{k}$.

(i) Let $0<C \leq 1$. The basis $\left(e_{k}\right)_{k}$ is called $C$-perturbable if whenever $T$ : $X \rightarrow X$ is a bounded linear operator for which there exists $\delta>0$ with $\left\|T\left(e_{k}\right)-e_{k}\right\|<C-\delta$ for all $k \in \mathbb{N}$, then $T(X)$ contains a copy of $X$.

(ii) If an operator $T$ on $X$ satisfies $\inf _{k}\left|e_{k}^{*}\left(T\left(e_{k}\right)\right)\right|>0$, then we say that $T$ has large diagonal.

(iii) An operator $T$ on $X$ satisfying $e_{m}^{*}\left(T\left(e_{k}\right)\right)=0$ whenever $k \neq m$, is called diagonal operator.

(iv) We say that the basis $\left(e_{k}\right)_{k}$ has the factorization property if whenever $T$ : $X \rightarrow X$ is a bounded linear operator with $\inf _{k}\left|e_{k}^{*}\left(T e_{k}\right)\right|>0$ then the identity of $X$ factors through $T$.

Remark 2.4. It is easy to see that a basis that has the factorizing property is also 1-perturbable. However, there are bases that are $C$-perturbable without the factorization property, as the following example shows.

The norm on the boundedly complete basis of James space $\left(e_{i}\right)_{i}$ is defined as follows:

$$
\left\|\sum_{i=1}^{n} a_{i} e_{i}\right\|=\sup \left(\sum_{k=1}^{m}\left(\sum_{i \in E_{k}} a_{i}\right)^{2}\right)^{1 / 2}
$$

where the supremum is taken over $m \in \mathbb{N}$ and sequences of successive intervals $\left(E_{k}\right)_{k=1}^{m}$ of natural numbers. Let $J$ denote the completion of the linear span of $\left(e_{i}\right)_{i}$ with this norm. Some well known important properties of $J$ are the following:

(i) The basis $\left(e_{i}\right)_{i}$ is spreading. In particular for any sequence scalars $\left(a_{i}\right)_{i=1}^{n}$ and natural numbers $k_{1}<\cdots<k_{n}$ we have

$$
\left\|\sum_{i=1}^{n} a_{i} e_{i}\right\|=\left\|\sum_{i=1}^{n} a_{i} e_{k_{i}}\right\| .
$$


(ii) The sequence $\left(e_{i}\right)_{i}$ is non-trivial weak Cauchy, i.e., there is $e^{* *} \in J^{* *} \backslash J$ so that $w^{*}-\lim _{i} e_{i}=e^{* *}$. Additionally, $\operatorname{dist}\left(e^{* *}, J\right)=1$.

(iii) The space $J$ is quasi-reflexive of order one. In particular, $J^{* *}=\mathbb{R} e^{* *} \oplus J$, i.e., $J^{* *}$ is spanned by $e^{* *}$ and the canonical embedding of $J$ in $J^{* *}$.

Note that by (iii) if the identity factors through an operator on $J$ then that operator cannot be weakly compact.

Proposition 2.5. There exists a weakly compact operator $T: J \rightarrow J$ with $e_{i}^{*}\left(T\left(e_{i}\right)\right)=$ 1 , for all $i \in \mathbb{N}$. In particular, $\left(e_{i}\right)$ dos not have the factorization property in $J$.

Proof. By (ii) the operator $S: J \rightarrow J$ given by $S e_{i}=e_{i+1}$, for all $i \in \mathbb{N}$, is a linear isometry. We define $T=I-S$, which has norm at most two. We will show that $S$ is weakly compact by showing that for every bounded sequence $\left(x_{i}\right)_{i}$ the sequence $\left(T x_{i}\right)_{i}$ has a weakly convergent subsequence. By the separability of $J^{*}$, we pass to a subsequence so that $\left(x_{i}\right)_{i}$ converges in the $w^{*}$-topology to some $x^{* *} \in J^{* *}$. By (iii) there is $x \in J$ and $c \in \mathbb{R}$ so that $x^{* *}=x+c e^{* *}$. We have

$$
\begin{aligned}
S^{* *}\left(x^{* *}\right) & =S(x)+c S^{* *}\left(e^{* *}\right)=S(x)+c\left(w^{*}-\lim _{i} S\left(e_{i}\right)\right) \\
& =S(x)+c\left(w^{*}-\lim _{i} e_{i+1}\right)=S(x)+c e^{* *} .
\end{aligned}
$$

Thus $w^{*}-\lim _{i} T x_{i}=w^{*}-\lim _{i}\left(x_{i}-S\left(x_{i}\right)\right)=x+c e^{* *}-\left(S(x)+c e^{* *}\right)=x-S(x)$. Because the $w^{*}$-limit is in $J$ it has to be a weak limit.

We wish to show now that the boundedly complete basis of James space is perturbable. To achieve that we shall need the following well known fact. We describe a proof for completeness.

Proposition 2.6. Let $\left(x_{i}\right)_{i}$ be a non-trivial weak Cauchy sequence in $J$. Then, $\left(x_{i}\right)_{i}$ has a subsequence $\left(x_{j_{i}}\right)_{i}$ that is equivalent to $\left(e_{i}\right)_{i}$ so that there exists a bounded linear projection $P: J \rightarrow\left[\left(x_{j_{i}}\right)_{i}\right]$.

Proof. Proposition 7.4 from [3] says the result holds, provided that the sequence $\left(e_{i}\right)_{i}$ is equivalent to its convex block sequences and not equivalent to the summing basis of $c_{0}$. Both of these properties follow from (1).

Proposition 2.7. Let $T: J \rightarrow J$ be a bounded linear operator with the property $\sup _{i}\left\|T e_{i}-e_{i}\right\|<1$. Then the identity factors through $T$. That is, the boundedly complete basis of $J$ is perturbable.

Proof. If $C=\liminf \left\|T\left(e_{i}\right)-e_{i}\right\|<1$ then we have $\left\|T^{* *}\left(e^{* *}\right)-e^{* *}\right\| \leq C$ and therefore, from (ii) $\operatorname{dist}\left(T^{* *}\left(e^{* *}\right), J\right) \geq 1-C>0$. This means that $T^{* *}\left(e^{* *}\right)$, which is the $w^{*}$-limit of $\left(T\left(e_{i}\right)\right)_{i}$, is not in $J$. In other words, $\left(T\left(e_{i}\right)\right)_{i}$ is non-trivial weak Cauchy. By Proposition 2.6 there is a subsequence $\left(T\left(e_{j_{i}}\right)\right)_{i}$ of $\left(T\left(e_{i}\right)\right)_{i}$ that is equivalent to $\left(e_{i}\right)_{i}$ and a bounded linear projection $P: J \rightarrow W=\left[\left(T\left(e_{j_{i}}\right)\right)_{i}\right]$. Let $A: J \rightarrow J$ be the map defined by $A e_{i}=e_{j_{i}}$, which by (i) is bounded. Let $R: W \rightarrow J$ be the isomorphism given by $R\left(T\left(e_{j_{i}}\right)\right)=e_{i}$ and set $B: J \rightarrow J$ with $B=R \circ P$. It is easy to see that $I=B \circ T \circ A$.

\section{Strategical Reproducibility, a condition implying the FACTORIZATION PROPERTY}

In this section we formulate several versions of a property of bases we call strategical reproducibility and show that they imply the factorization property. 
Notation and conventions. All our Banach spaces are assumed to be over the real numbers $\mathbb{R}$. $B_{X}$ denotes the unit ball, and $S_{X}$ denotes the unit sphere of a Banach space $X$. $c_{00}$ denotes the sequences in $\mathbb{R}$ which eventually vanish.

For a Banach space $X$ we denote by $\operatorname{cof}(X)$ the set of cofinite dimensional subspaces of $X$, while $\operatorname{cof}_{w^{*}}\left(X^{*}\right)$ denotes the cofinite dimensionl $w^{*}$-closed subspaces of $X^{*}$.

If $\bar{e}=\left(e_{i}\right)$ is a basis of a Banach space $X$, we call for $x=\sum_{i=1}^{\infty} x_{i} e_{i} \in X$ the set $\left\{i \in \mathbb{N}: x_{i} \neq 0\right\}$ the support of $x$ with respect to $(\bar{e})$ and denote it by $\operatorname{supp}_{\bar{e}}(x)$. If there is no confusion possible, we also may write $\operatorname{supp}(x)$ instead of $\operatorname{supp}_{\bar{e}}(x)$.

We recall that a basis $\left(e_{n}\right)$ of a Banach space $X$ is shrinking if the coordinate functionals $\left(e_{n}^{*}\right)$ are a basis of $X^{*}$, and unconditional if for some constant $c \geq 1$ and all finite sequence of scalars $\left(a_{i}\right)_{i=1}^{n}$, and all $\sigma=\left(\sigma_{i}\right)_{i=1}^{n} \in\{ \pm 1\}$

$$
\left\|\sum_{i=1}^{n} \sigma_{i} a_{i} e_{i}\right\| \leq c\left\|\sum_{i=1}^{n} a_{i} e_{i}\right\|
$$

Let $\left(x_{i}\right)_{i}$ and $\left(y_{i}\right)_{i}$ be Schauder basic sequences in (possibly different) Banach spaces and $C \geq 1$. We say that $\left(x_{i}\right)_{i}$ and $\left(y_{i}\right)_{i}$ are $C$-equivalent if there are $A, B>0$, with $A \cdot B \leq C$ so that for any $\left(a_{i}\right)_{i} \in c_{00}$ of scalars we have

$$
\frac{1}{A}\left\|\sum_{i=1}^{\infty} a_{i} y_{i}\right\| \leq\left\|\sum_{i=1}^{\infty} a_{i} x_{i}\right\| \leq B\left\|\sum_{i=1}^{\infty} a_{i} y_{i}\right\| .
$$

We say that $\left(x_{i}\right)_{i}$ and $\left(y_{i}\right)_{i}$ are "impartially $C$-equivalent" if for any finite choice of scalars $\left(a_{i}\right)_{i} \in c_{00}$ we have

$$
\frac{1}{\sqrt{C}}\left\|\sum_{i=1}^{\infty} a_{i} y_{i}\right\| \leq\left\|\sum_{i=1}^{\infty} a_{i} x_{i}\right\| \leq \sqrt{C}\left\|\sum_{i=1}^{\infty} a_{i} y_{i}\right\| .
$$

Note that if two sequences are $C$-equivalent then by scaling one of them we can always make them impartially $C$-equivalent.

We now formally define the concept of strategical reproducibility depending on properties of the basis of a Banach space. The most general form will be given in Defitinion 3.4. Nevertheless, under additional assumptions on the basis, this notion considerably simplifies. The proof that the different definitions of strategical reproducibility are equivalent under their respective assumptions on the basis will be given later.

If we demand that our basis is unconditional and shrinking strategical reproducibility can be defined as follows.

Definition 3.1. Assume that $X$ is a Banach space with a basis $\left(e_{n}\right)$ which is unconditional and shrinking. Let $\left(e_{n}^{*}\right) \subset X^{*}$ be the corresponding coordinate functionals. We say that $\left(e_{n}\right)$ is strategically reproducible if the following condition is satisfied for some $C \geq 1$ :

$$
\begin{aligned}
& \forall n_{1} \in \mathbb{N} \exists b_{1} \in \operatorname{span}\left(e_{n}: n \geq n_{1}\right) \exists b_{1}^{*} \in \operatorname{span}\left(e_{n}^{*}: n \geq n_{1}\right) \\
& \forall n_{2} \in \mathbb{N} \exists b_{2} \in \operatorname{span}\left(e_{n}: n \geq n_{2}\right) \exists b_{2}^{*} \in \operatorname{span}\left(e_{n}^{*}: n \geq n_{2}\right) \\
& \forall n_{3} \in \mathbb{N} \exists b_{3} \in \operatorname{span}\left(e_{n}: n \geq n_{3}\right) \exists b_{3}^{*} \in \operatorname{span}\left(e_{n}^{*}: n \geq n_{3}\right) \\
& \vdots
\end{aligned}
$$


so that:

(3a) $\left(b_{k}\right)$ is impartially $C$-equivalent to $\left(e_{k}\right)$, and

$\left(b_{k}^{*}\right)$ is impartially $C$-equivalent to $\left(e_{k}^{*}\right)$,

(3p) $b_{k}^{*}\left(b_{l}\right)=\delta_{k, l}$ for all $k, l \in \mathbb{N}$.

Remark 3.2. Condition (3) in Definition 3.1 can be interpreted that one player in a two-person game has a winning strategy:

We fix $C \geq 1$. Player I chooses $n_{1} \in \mathbb{N}$, then player (II) chooses $b_{1} \in \operatorname{span}\left(e_{n}\right.$ : $\left.n \geq n_{1}\right)$ and $b_{1}^{*} \in \operatorname{span}\left(e_{n}^{*}: n \geq n_{1}\right)$. They repeat the moves infinitely many times, obtaining for every $k \in \mathbb{N}$ numbers $n_{k}$, and vectors $b_{k}$ and $b_{k}^{*}$. Player II wins if he was able to choose the sequences $\left(b_{n}\right) \subset X$ and $\left(b_{n}^{*}\right) \subset X^{*}$ so that $(3 \mathrm{a}),(3 \mathrm{~b})$ are satisfied. Thus, the basis $\left(e_{i}\right)$ is strategically reproducible if and only if for some $C \geq 1$ player (II) has a winning strategy.

In general it is not true that two-player games of infinite length are determined, i.e., that one of the player has a winning strategy. Nevertheless, for $C \geq 1$ it is easy to see that the set of all sequences $\left(b_{k}, b_{k}^{*}\right)$ in $\left(X \times X^{*}\right)^{\mathbb{N}}$ which satisfy $3(\mathrm{a})$ and $3(\mathrm{~b})$ is Borel measurable (it is actually closed) with respect to the product topology of the discrete topology on $X \times X^{*}$, and thus it follows from the main result in 15 that this game is determined. More on these Infinite Asymptotic Games can be found in $[22$.

Now we relax the condition on our basis $\left(e_{i}\right)$ and only require it be unconditional. In that case we define strategical reproducible as follows.

Definition 3.3. Let $X$ be a Banach space with an unconditional basis $\left(e_{i}\right)_{i}$ and fix positive constants $C \geq 1$.

Consider the following two-player game between player (I) and player (II). For $k \in \mathbb{N}$, turn $k$ is played out in three steps.

Step 1: Player (I) chooses $\eta_{k}>0, W_{k} \in \operatorname{cof}(X)$, and $G_{k} \in \operatorname{cof}_{w^{*}}\left(X^{*}\right)$,

Step 2: Player (II) chooses a finite subset $E_{k}$ of $\mathbb{N}$ and sequences of non-negative real numbers $\left(\lambda_{i}^{k}\right)_{i \in E_{k}},\left(\mu_{i}^{k}\right)_{i \in E_{k}}$ satisfying

$$
\sum_{i \in E_{k}} \lambda_{i}^{(k)} \mu_{i}^{(k)}=1 .
$$

Step 3: Player (I) chooses $\left(\varepsilon_{i}^{(k)}\right)_{n \in E_{k}}$ in $\{-1,1\}^{E_{k}}$.

We say that player (II) has a winning strategy in the game $\operatorname{Rep}_{\left(X,\left(e_{i}\right)\right)}(C)$ if he can force the following properties on the result:

For all $n \in \mathbb{N}$ we set $x_{k}=\sum_{i \in E_{k}} \varepsilon_{i}^{(k)} \lambda_{i}^{(k)} e_{i}$ and $x_{k}^{*}=\sum_{i \in E_{k}} \varepsilon_{i}^{(k)} \mu_{i}^{(k)} e_{i}^{*}$ and demand:

(i) the sequences $\left(x_{k}\right)_{k}$ and $\left(e_{k}\right)_{k}$ are impartially $C$-equivalent,

(ii) the sequences $\left(x_{k}^{*}\right)_{k}$ and $\left(e_{k}^{*}\right)_{k}$ are impartially $C$-equivalent,

(iii) for all $n \in \mathbb{N}$ we have $\operatorname{dist}\left(x_{k}, W_{k}\right)<\eta_{k}$, and

(iv) for all $n \in \mathbb{N}$ we have $\operatorname{dist}\left(x_{k}^{*}, G_{k}\right)<\eta_{k}$.

We say that $\left(e_{i}\right)_{i}$ is $C$-strategically reproducible in $X$ if for every $\eta>0$ player II has a winning strategy in the game $\operatorname{Rep}_{\left(X,\left(e_{i}\right)\right)}(C+\eta)$.

Finally we will not even require the basis $\left(e_{j}\right)$ to be unconditional and define strategical reproducible as follows: 
Definition 3.4. Let $X$ be a Banach space with a normalized Schauder basis $\left(e_{i}\right)_{i}$ and fix positive constants $C \geq 1$, and $\eta>0$.

Consider the following two-player game between player (I) and player (II):

Before the first turn player (I) is allowed to choose a partition of $\mathbb{N}=N_{1} \cup N_{2}$. For $k \in \mathbb{N}$, turn $k$ is played out in three steps.

Step 1: Player (I) chooses $\eta_{k}>0, W_{k} \in \operatorname{cof}(X)$, and $G_{k} \in \operatorname{cof}_{w^{*}}\left(X^{*}\right)$, Step 2: Player (II) chooses $i_{k} \in\{1,2\}$, a finite subset $E_{k}$ of $N_{i_{k}}$ and sequences of non-negative real numbers $\left(\lambda_{i}^{k}\right)_{i \in E_{k}},\left(\mu_{i}^{k}\right)_{i \in E_{k}}$ satisfying

$$
1-\eta<\sum_{i \in E_{k}} \lambda_{i}^{(k)} \mu_{i}^{(k)}<1+\eta .
$$

Step 3: Player (I) chooses $\left(\varepsilon_{i}^{(k)}\right)_{n \in E_{k}}$ in $\{-1,1\}^{E_{k}}$.

We say that player (II) has a winning strategy in the game $\operatorname{Rep}_{\left(X,\left(e_{i}\right)\right)}(C, \eta)$ if he can force the following properties on the result:

For all $n \in \mathbb{N}$ we set $x_{k}=\sum_{i \in E_{k}} \varepsilon_{i}^{(k)} \lambda_{i}^{(k)} e_{i}$ and $x_{k}^{*}=\sum_{i \in E_{k}} \varepsilon_{i}^{(k)} \mu_{i}^{(k)} e_{i}^{*}$ and demand:

(i) the sequences $\left(x_{k}\right)_{k}$ and $\left(e_{k}\right)_{k}$ are impartially $(C+\eta)$-equivalent,

(ii) the sequences $\left(x_{k}^{*}\right)_{k}$ and $\left(e_{k}^{*}\right)_{k}$ are impartially $(C+\eta)$-equivalent,

(iii) for all $n \in \mathbb{N}$ we have $\operatorname{dist}\left(x_{k}, W_{k}\right)<\eta_{k}$, and

(iv) for all $n \in \mathbb{N}$ we have $\operatorname{dist}\left(x_{k}^{*}, G_{k}\right)<\eta_{k}$.

We say that $\left(e_{i}\right)_{i}$ is $C$-strategically reproducible in $X$ if for every $\eta>0$ player II has a winning strategy in the game $\operatorname{Rep}_{\left(X,\left(e_{i}\right)\right)}(C, \eta)$.

Remark 3.5. We first want to observe that if $\left(e_{i}\right)$ is a normalized shrinking and unconditional basis then being strategically reproducible in the sense of Definition 3.1 is equivalent with being $C$-strategically reproducible for some $C \geq 1$ in the sense of Definition 3.4 .

Indeed, assume $\left(e_{i}\right)$ is 1-unconditional and shrinking and assume that for some $\tilde{C} \geq 1 \sqrt{3})$ of Definition 3.1 holds. We will show that $\left(e_{i}\right)$ is $3 \tilde{C}$-strategically reproducible in the sense of Definition 3.4 .

Let $1 / 3>\eta>0$ be given and assume player (I) has at the beginning of the game chosen a partition $\left(N_{1}, N_{2}\right)$ of $\mathbb{N}$. At the $k$-th step player (I) chooses $\eta_{k}>0$ and spaces $W_{k} \in \operatorname{cof}(X)$ and $G_{k} \in \operatorname{cof}_{w^{*}}(X)$. Since $\left(e_{k}\right)$ is shrinking, player (II) can "approximate $W_{k}$ by a tail space" as follows: there is $n_{k}^{(1)} \in \mathbb{N}$ so that for all $x \in B_{X} \cap\left[e_{i}: i \geq n_{k}^{(1)}\right]$ it follows that $\operatorname{dist}\left(x, W_{k}\right)<\eta_{k} / 2 \tilde{C}$. Secondly, since $G_{k}$ is a $w^{*}$-closed and cofinite dimensional subspace of $X^{*}$, and thus the annihilator of a finite subset of $X$, we find $n_{k}^{(2)} \in \mathbb{N}$ so that for all $x^{*} \in B_{X^{*}} \cap\left[e_{i}^{*}: i \geq n_{k}^{(2)}\right]$ it follows that $\operatorname{dist}\left(x^{*}, W_{k}\right)<\eta_{k} / 2 \tilde{C}$. Finally we let $n_{k}^{(3)}=1+\max \left(\bigcup_{j=1}^{k-1} \operatorname{supp}\left(x_{j}\right) \cup\right.$ $\left.\operatorname{supp}\left(x_{j}^{*}\right)\right)$. Let $n_{k}=\max \left(n_{k}^{(1)}, n_{k}^{(2)}, n_{k}^{(3)}\right)$ and let player (II) follow his winning strategy, assuming player (I) has chosen $n_{k} \in \mathbb{N}$ in his $k$-th move of the game described in Definition 3.1, and let $b_{k} \in\left[e_{i}: i \geq n_{k}\right]$ and $b_{k}^{*} \in\left[e_{i}^{*}: i \geq n_{k}\right]$ be chosen according to that strategy, which in particular implies that $\left\|b_{k}\right\|,\left\|b_{k}^{*}\right\| \leq \sqrt{\tilde{C}}$. We write $b_{k}$ and $b_{k}^{*}$ as

$$
b_{k}=\sum_{j=1}^{\infty} \tilde{\lambda}_{j}^{(k)} e_{j} \text { and } b_{k}^{*}=\sum_{j=1}^{\infty} \tilde{\mu}_{j}^{(k)} e_{j} .
$$


By reducing the supports, if necessary we can assume, by using Proposition 3.8 . that $\tilde{E}_{k}=\operatorname{supp}\left(b_{k}\right)=\operatorname{supp}\left(b_{k}^{*}\right)$ and since $b_{k}^{*}\left(b_{k}\right)=\sum_{j=1}^{\infty} \tilde{\mu}_{j}^{(k)} \tilde{\lambda}_{j}^{(k)}=1$ we can choose $i_{k} \in\{1,2\}$, so that

$$
\rho_{k}=\sum_{j \in N_{i_{k}}} \tilde{\mu}_{j}^{(k)} \tilde{\lambda}_{j}^{(k)} \geq \frac{1}{2}
$$

Then we let $E_{k}=\tilde{E}_{k} \cap N_{i_{k}}, \mu_{j}^{(k)}=\tilde{\mu}_{j}^{(k)} / \sqrt{\rho_{k}}$ and $\lambda_{j}^{(k)}=\tilde{\lambda}_{j}^{(k)} / \sqrt{\rho_{k}}$ for $j \in E_{k}$. After player (I) has chosen $\left(\varepsilon_{j}^{(k)}\right)_{j \in E_{k}}$ we also put $x_{k}=\sum_{j \in E_{k}} \varepsilon_{j}^{(k)} \mu_{j}^{(k)} e_{j} \in \sqrt{2 \tilde{C}} B_{X} \cap\left[e_{j}\right.$ : $\left.j \geq n_{k}\right]$ and $x_{k}^{*}=\sum_{j \in E_{k}} \varepsilon_{j}^{(k)} \lambda_{j}^{(k)} e_{j}^{*} \in \sqrt{2 \tilde{C}} B_{X^{*}} \cap\left[e_{i}^{*}: i \geq n_{k}\right]$.

From the choice of $n_{k}$, and the fact that $\left\|x_{k}\right\| \leq \sqrt{2 \tilde{C}}$ and $\left\|x_{k}^{*}\right\| \leq \sqrt{2 \tilde{C}}$, it follows that $\operatorname{dist}\left(x_{k}, W_{k}\right)<\eta_{k}$ and $\operatorname{dist}\left(x^{*}, G_{k}\right)<\eta_{k}$. From the 1-unconditionality of $\left(e_{j}\right)$ it follows for $\left(\xi_{k}\right) \in c_{00}$ that

$$
\left\|\sum_{k=1}^{\infty} \xi_{k} x_{k}\right\| \leq \sqrt{2}\left\|\sum_{k=1}^{\infty} \xi_{k} b_{k}\right\| \leq \sqrt{2} \sqrt{\tilde{C}} \leq\left\|\sum_{k=1}^{\infty} \xi_{k} e_{k}\right\|
$$

and

$$
\left\|\sum_{k=1}^{\infty} \xi_{k} x_{k}^{*}\right\| \leq \sqrt{2}\left\|\sum_{k=1}^{\infty} \xi_{k} b_{k}^{*}\right\| \leq \sqrt{2} \sqrt{\tilde{C}} \leq\left\|\sum_{k=1}^{\infty} \xi_{k} e_{k}^{*}\right\| .
$$

Thus, by Proposition 3.8 below, $\left(x_{k}\right)$ is impartially $2 \tilde{C}$-equivalent to $\left(e_{k}\right)$ and $\left(e_{k}^{*}\right)$ is impartially $2 \tilde{C}$-equivalent to $\left(e_{k}^{*}\right)$.

Conversely, it is easy to deduce that if $\left(e_{j}\right)$ is unconditional and shrinking and strategically reproducible in the sense of Definition 3.4. then it is also strategically reproducible in the sense of Definition 3.1 .

In a similar way we can show that for an unconditional and normalized basis $\left(e_{j}\right)$ strategical reproducibility in sense of Definitions 3.3 and 3.4 are equivalent.

Remark 3.6. The unit vector basis of $\ell_{1}$ has the factorization property yet it is not strategically reproducible under any of the above definitions. It is possible to give a fourth notion of strategic reproducibility that covers $\ell_{1}$, is strictly less restrictive than Definition 3.4, and implies the factorization property. This formulation is rather technical, so we will not discuss it in the present paper.

We will now show that a basis which is strategically reproducible has the factorization property. We will first need the following two observations.

Lemma 3.7. Assume that $X$ is a Banach space with a basis $\left(e_{n}\right)$, whose basis constant is $\lambda \geq 1$ and biorthogonal functionals $\left(e_{n}^{*}\right)$. Let $\left(b_{n}\right)$ and $\left(b_{n}^{*}\right)$ be block bases of $\left(e_{n}\right)$ and $\left(e_{n}^{*}\right)$, respectively, so that $b_{m}^{*}\left(b_{n}\right)=\delta_{m, n}$, for $m, n \in \mathbb{N}$, and so that for some $C \geq 1$ it follows that

$$
\left\|\sum_{j=1}^{\infty} \xi_{j} b_{j}\right\|_{X} \leq \sqrt{C}\left\|\sum_{j=1}^{\infty} \xi_{j} e_{j}\right\|_{X} \quad \text { and } \quad\left\|\sum_{j=1}^{\infty} \xi_{j} b_{j}^{*}\right\|_{X^{*}} \leq \sqrt{C}\left\|\sum_{j=1}^{\infty} \xi_{j} e_{j}^{*}\right\|_{X^{*}},
$$

for all $\left(\xi_{j}\right) \in c_{00}$.

Then $Y=\overline{\operatorname{span}\left(b_{j}: j \in \mathbb{N}\right)}$ is a complemented subspace of $X$ and

$$
P: X \rightarrow Y, \quad x \mapsto \sum_{n=1}^{\infty} b_{n}^{*}(x) b_{n}
$$


is well defined and a bounded projection onto $Y$ with $\|P\| \leq \lambda C$. Moreover, if $\left(e_{n}\right)$ is shrinking, then $\|P\| \leq C$.

Proof. If $\left(e_{n}\right)$ is shrinking then $\operatorname{span}\left(e_{n}^{*}: n \in \mathbb{N}\right)$ is norm dense in $X^{*}$ and therefore we have in that case

$$
\|x\|=\sup _{x^{*} \in \operatorname{span}\left(e_{n}^{*}: n \in \mathbb{N}\right)} x^{*}(x) .
$$

If $\left(e_{n}\right)$ is a general basis whose basis constant is $\lambda$, we denote by $P_{n}$ the projection $P_{n}: X \rightarrow X, \sum_{j=1}^{\infty} x_{j} e_{j} \mapsto \sum_{j=1}^{n} x_{j} e_{j}$, and since $\left\|P_{n}\right\|=\left\|P_{n}^{*}\right\| \leq \lambda$ we deduce for $x \in X$

$$
\begin{aligned}
\|x\| & =\lim _{n \rightarrow \infty} \sup _{x^{*} \in B_{X^{*}}} x^{*}\left(P_{n}(x)\right) \leq \sup _{n \in \mathbb{N}, x^{*} \in B_{X^{*}}} P_{n}^{*}\left(x^{*}\right)(x) \\
& \leq \sup _{z^{*} \in \lambda B_{X^{*}} \cap \operatorname{nppan}\left(e_{j}^{*}: j \in \mathbb{N}\right)} z^{*}(x)=\lambda z_{z^{*} \in B_{X^{*}} \cap \operatorname{npan}\left(e_{j}^{*}: j \in \mathbb{N}\right)} z^{*}(x) .
\end{aligned}
$$

If $x \in \operatorname{span}\left(e_{j}\right)$ then $P(x)$ is a finite linear combination of elements of $\left(b_{n}\right)$.

We compute:

$$
\begin{aligned}
\sup _{x \in B_{X} \cap \operatorname{span}\left(e_{j}: j \in \mathbb{N}\right)}\|P(x)\| & =\sup _{x \in B_{X} \cap \operatorname{span}\left(e_{j}: j \in \mathbb{N}\right)}\left\|\sum_{j=1}^{\infty} b_{j}^{*}(x) b_{j}\right\| \\
& \leq \sqrt{C} \sup _{x \in B_{X} \cap \operatorname{span}\left(e_{j}: j \in \mathbb{N}\right)}\left\|\sum_{j=1}^{\infty} b_{j}^{*}(x) e_{j}\right\| .
\end{aligned}
$$

Using (5) yields

$$
\begin{aligned}
\sup _{x \in B_{X} \cap \operatorname{span}\left(e_{j}: j \in \mathbb{N}\right)}\|P(x)\| & \leq \lambda \sqrt{C} \sup _{\substack{x \in B_{X} \cap \operatorname{span}\left(e_{j}: j \in \mathbb{N}\right) \\
x^{*} \in B_{X^{*}} \cap \operatorname{span}\left(e_{j}^{*}: j \in \mathbb{N}\right)}} x^{*}\left(\sum_{j=1}^{\infty} b_{j}^{*}(x) e_{j}\right) \\
& =\lambda \sqrt{C} \sup _{x^{*} \in B_{X^{*}} \cap \operatorname{span}\left(e_{j}^{*}: j \in \mathbb{N}\right)}\left\|\sum_{j=1}^{\infty} x^{*}\left(e_{j}\right) b_{j}^{*}\right\| .
\end{aligned}
$$

By (4), we obtain therefore

$$
\sup _{x \in B_{X} \cap \operatorname{span}\left(e_{j}: j \in \mathbb{N}\right)}\|P(x)\| \leq \lambda \sqrt{C} \sup _{\left(\xi_{j}\right) \in c_{00},\left\|\sum \xi_{j} e_{j}^{*}\right\| \leq 1}\left\|\sum_{j=1}^{\infty} \xi_{j} b_{j}^{*}\right\| \leq \lambda C .
$$

In the case that $\left(e_{j}\right)$ is shrinking we can replace in the first inequality $\lambda$ by 1 , and therefore obtain that $\|P(x)\| \leq C$ for $x \in B_{X}$.

Proposition 3.8. Assume that $X$ is a Banach space with a basis $\left(e_{n}\right)$ and biorthogonal functionals $\left(e_{n}^{*}\right)$. Let $\left(b_{n}\right)$ and $\left(b_{n}^{*}\right)$ be block bases of $\left(e_{n}\right)$ and $\left(e_{n}^{*}\right)$, respectively, so that $b_{m}^{*}\left(b_{n}\right)=\delta_{m, n}$, for $m, n \in \mathbb{N}$, and so that for some $C \geq 1$ it follows that

$$
\left\|\sum_{j=1}^{\infty} \xi_{j} b_{j}\right\|_{X} \leq \sqrt{C}\left\|\sum_{j=1}^{\infty} \xi_{j} e_{j}\right\|_{X} \quad \text { and } \quad\left\|\sum_{j=1}^{\infty} \xi_{j} b_{j}^{*}\right\|_{X^{*}} \leq \sqrt{C}\left\|\sum_{j=1}^{\infty} \xi_{j} e_{j}^{*}\right\|_{X^{*}}
$$

for all $\left(\xi_{j}\right) \in c_{00}$.

Then $\left(b_{n}\right)$ is $\lambda C$-impartially equivalent to $\left(e_{n}\right)$ and $\left(b_{n}^{*}\right)$ is $C$-impartially equivalent to $\left(e_{n}\right)$. If $\left(e_{n}\right)_{n}$ is Shrinking then $\left(b_{n}\right)$ is $C$-impartially equivalent to $\left(e_{n}\right)$. 
Proof. For a sequence $\left(\xi_{j}\right) \in c_{00}$ we compute

$$
\begin{aligned}
\left\|\sum_{j=1}^{\infty} \xi_{j} b_{j}\right\| & \geq \sup _{\left(\eta_{j}\right) \in c_{00},\left\|\sum_{j=1}^{\infty} \eta_{j} b_{j}^{*}\right\| \leq 1} \sum_{j=1}^{\infty} \eta_{j} b_{j}^{*}\left(\sum_{j=1}^{\infty} \xi_{j} b_{j}\right) \\
& =\sup _{\left(\eta_{j}\right) \in c_{00},\left\|\sum_{j=1}^{\infty} \eta_{j} b_{j}^{*}\right\| \leq 1} \sum_{j=1}^{\infty} \xi_{j} \cdot \eta_{j} .
\end{aligned}
$$

By (6) and then (5) we obtain

$$
\begin{aligned}
\left\|\sum_{j=1}^{\infty} \xi_{j} b_{j}\right\| & \geq \sup _{\left(\eta_{j}\right) \in c_{00},\left\|\sum_{j=1}^{\infty} \eta_{j} e_{j}^{*}\right\| \leq 1 / \sqrt{C}} \sum_{j=1}^{\infty} \xi_{j} \cdot \eta_{j} \\
& =\frac{1}{\sqrt{C}} \sup _{\left(\eta_{j}\right) \in c_{00},\left\|\sum_{j=1}^{\infty} \eta_{j} e_{j}^{*}\right\| \leq 1} \sum_{j=1}^{\infty} \xi_{j} \cdot \eta_{j}=\geq \frac{1}{\lambda \sqrt{C}}\left\|\sum_{j=1}^{\infty} \xi_{j} e_{j}\right\| .
\end{aligned}
$$

Similarly we show that

$$
\left\|\sum_{j=1}^{\infty} \xi_{j} b_{j}^{*}\right\| \geq \frac{1}{\sqrt{C}}\left\|\sum_{j=1}^{\infty} \xi_{j} e_{j}^{*}\right\| .
$$

In order to deduce that strategical reproducibility implies the factorization property, we will also need a condition on diagonal operators which is automatically satisfied in the case that the given basis is unconditional.

Definition 3.9. Let $X$ be a Banach space with a normalized Schauder basis $\left(e_{n}\right)_{n}$. We say that the basis $\left(e_{n}\right)_{n}$ has the uniform diagonal factorization property if for every $\delta>0$ there exists $K(\delta) \geq 1$ so that for every bounded diagonal operator $T: X \rightarrow X$ with $\inf _{n}\left|e_{n}^{*}\left(T\left(e_{n}\right)\right)\right| \geq \delta$ the identity almost $K(\delta)$-factors through $T$. If we wish to be more specific we shall say that $\left(e_{n}\right)_{n}$ has the $K(\delta)$-diagonal factorization property.

Note that if $\left(e_{n}\right)$ is unconditional then it has the uniform diagonal factorization property. The following definition quantifies the uniform factorization property.

Definition 3.10. Let $X$ be a Banach space with a normalized Schauder basis $\left(e_{n}\right)_{n}$. We say that the basis $\left(e_{n}\right)_{n}$ has the uniform factorization property if for every $\delta>0$ there exists $K(\delta) \geq 1$ so that for every bounded linear operator $T: X \rightarrow X$ with $\inf _{n}\left|e_{n}^{*}\left(T\left(e_{n}\right)\right)\right| \geq \delta$ the identity almost $K(\delta)$-factors through $T$. If we wish to be more specific we shall say that $\left(e_{n}\right)_{n}$ has the $K(\delta)$-factorization property.

Remark 3.11. Note that in Definitions 3.9 and $3.10 K(\delta) \geq 1 / \delta$. This can be witnessed by taking $T=\delta I$. Also whenever the function $K:(0, \infty) \rightarrow \mathbb{R}$ is well defined it is also continuous. In fact, for $0<\varepsilon<\delta$ if a simple scaling argument yields

$$
K(\delta) \leq K(\delta-\varepsilon) \leq \frac{\delta}{\delta-\varepsilon} K(\delta) .
$$

To see this, use the following trick: if $T$ has a diagonal whose elements are absolutey bounded below by $\delta-\varepsilon$ then the elements of the diagonal of $\delta /(\delta-\varepsilon) T$ are absolutey bounded below by $\delta$. Inspecting (7) we also deduce $K(\delta) \leq K(1) / \delta$ and therefore

for all $\delta>0$.

$$
\frac{1}{\delta} \leq K(\delta) \leq \frac{K(1)}{\delta}
$$


Theorem 3.12. Let $X$ be a Banach space with a Schauder basis $\left(e_{n}\right)_{n}$ that has a basis constant $\lambda$. Assume also that

(i) the basis $\left(e_{i}\right)_{i}$ has the $K(\delta)$-diagonal factorization property and

(ii) the basis $\left(e_{i}\right)_{i}$ is $C$-strategically reproducible in $X$.

Then $\left(e_{i}\right)_{i}$ has the $\lambda C^{2} K(\delta)$-factorization property.

Remark 3.13. It is worth pointing out that in Definition 3.10 the norm of $T$ does not appear and the factorization constant of the identity through $T$ depends only on the diagonal of $T$. This means that having the uniform factorization property is formally stronger than having the factorization property. Theorem 3.12 yields the stronger property. It is unclear whether these two properties are actually distinct.

We postpone the proof of Theorem 3.12 to first present a necessary lemma.

Lemma 3.14. Let $X$ be a Banach space with a normalized Schauder basis $\left(e_{n}\right)_{n}$ with a basis constant $\lambda$, let $T: X \rightarrow X$ be a bounded linear operator, let $\left(x_{n}\right)_{n}$, $\left(x_{n}^{*}\right)_{n}$ be sequences in $X$ and $X^{*}$ respectively and let $\eta>0, C \geq 1$. Assume that the following are satisfied:

(i) $\left(x_{n}\right)_{n}$ and $\left(e_{n}\right)_{n}$ are impartially $C$-equivalent,

(ii) $\left(x_{n}^{*}\right)_{n}$ and $\left(e_{n}^{*}\right)_{n}$ are impartially $C$-equivalent,

(iii) there exists a block sequence $\left(\tilde{x}_{n}^{*}\right)_{n}$ of $\left(e_{n}^{*}\right)$ so that $\sum_{n}\left\|x_{n}^{*}-\tilde{x}_{n}^{*}\right\|<\infty$,

(iv) $\sum_{n} \sum_{m \neq n}\left|x_{n}^{*}\left(T\left(x_{m}\right)\right)\right|<\eta$.

Then the diagonal operator $D: X \rightarrow X$ given by $D\left(e_{n}\right)=x_{n}^{*}\left(T\left(x_{n}\right)\right) e_{n}$ is bounded and there exist bounded linear operators $B, A: X \rightarrow X$ with $\|A\|\|B\| \leq \lambda C^{2}$ so that $\|D-B T A\|<2 \lambda \eta$.

If we additionally assume that $K \geq 1$ is such that the identity $K$-factors through $D$ and $\eta<1 /(2 \lambda K)$ then the identity $\left(\frac{K C^{2}}{1-2 \lambda K \eta}\right)$-factors through $T$.

Proof. The maps $A: X \rightarrow X, S:\left[x_{n}: n \in \mathbb{N}\right] \rightarrow X$ with $A\left(e_{n}\right)=x_{n}, S\left(x_{n}\right)=e_{n}$ are well defined and satisfy $\|A\|\|S\| \leq C$.

From Lemma 3.7 it follows that the map $R: X \rightarrow\left[x_{n}: n \in \mathbb{N}\right]$ given by $R(x)=\sum_{n=1}^{\infty} x_{n}^{*}(x) x_{n}$ is well defined and $\|R\| \leq \lambda C$.

Define $B=S \circ R: X \rightarrow X$. Then $\|B \circ A\| \leq \lambda C^{2}$. It also follows that for each $m \in \mathbb{N}$ we have $B T A\left(e_{m}\right)=\sum_{n=1}^{\infty} x_{n}^{*}\left(T\left(x_{m}\right)\right) e_{n}$. By (iv) we deduce that for each $m \in \mathbb{N}$ we have

$$
\left\|B T A\left(e_{m}\right)-x_{m}^{*}\left(T\left(x_{m}\right)\right) e_{m}\right\| \leq \sum_{n \neq m}\left|x_{n}^{*}\left(T\left(x_{m}\right)\right)\right| .
$$

Combining this with the triangle inequality we obtain that the desired diagonal map $D$ is bounded and $\|D-B T A\|<2 \lambda \eta$.

For the additional part, assume that $\hat{B}: X \rightarrow X$ and $\hat{A}: X \rightarrow X$ are such that $\|\hat{B}\|\|\hat{A}\| \leq K$ and $I=\hat{B} D \hat{A}$. It follows that $\|I-\hat{B} B T A \hat{A}\|=\|\hat{B}(D-B T A) \hat{A}\|<$ $2 \lambda K \eta<1$. Hence, the map $Q=\hat{B} B T A \hat{A}$ is invertible with $\left\|Q^{-1}\right\| \leq 1 /(1-2 \lambda K \eta)$. In conclusion, if we set $\tilde{B}=Q^{-1} \hat{B} B, \tilde{A}=A \hat{A}$ then $\tilde{B} T \tilde{A}=I$ and $\|\tilde{B}\|\|\tilde{A}\| \leq$ $\lambda K C^{2} /(1-2 \lambda K \eta)$.

Proof of Theorem 3.12, Let $\delta>0, T: X \rightarrow X$ be a bounded linear operator and $\inf _{n}\left|e_{n}^{*}\left(T\left(e_{n}\right)\right)\right| \geq \delta$. Let us fix $\eta>0$ to be determined later.

We will now describe a strategy for player (I) in a game $\operatorname{Rep}_{\left(X,\left(e_{i}\right)\right)}(C, \eta)$, and assume player (II) answers by following his winning strategy. 
At the beginning, as player (I) chooses $N_{1}=\left\{n \in \mathbb{N}: e_{n}^{*}\left(T\left(e_{n}\right)\right) \geq \delta\right\}$ and $N_{2}=\left\{n \in \mathbb{N}: e_{n}^{*}\left(T\left(e_{n}\right)\right) \leq-\delta\right\}$. In the first step of the $n$ 'th turn he chooses $\eta_{n}<\eta\left(\|T\| n 2^{n} \sqrt{C+\eta}\right)^{-1}$ and if $l_{n}=\max _{1 \leq k<n}\left(E_{k}\right)$ chooses $G_{n}=A_{n}^{\perp}$ and $W_{n}=$ $\left(B_{n}\right)_{\perp}$, where

$$
\begin{aligned}
A_{n} & =\left\{x_{1}, T\left(x_{1}\right), \ldots, x_{n-1}, T\left(x_{n-1}\right), e_{1}, T\left(e_{1}\right), \ldots, e_{l_{n}}, T\left(e_{l_{n}}\right)\right\}, \\
B_{n} & =\left\{x_{1}^{*}, T^{*}\left(x_{1}\right), \ldots, x_{n}^{*}, T^{*}\left(x_{n}^{*}\right), e_{1}^{*}, T^{*}\left(e_{1}^{*}\right), \ldots, e_{l_{n}}^{*}, T^{*}\left(e_{l_{n}}^{*}\right)\right\} .
\end{aligned}
$$

Player (II), following a winning strategy, chooses $i_{n}=1$ or $i_{n}=2$, picks $E_{n} \subset N_{i_{n}}$, and non-negative scalars $\left(\lambda_{i}^{(n)}\right)_{i \in E_{n}},\left(\mu_{i}^{(n)}\right)_{i \in E_{n}}$ with

$$
1-\eta<\sum_{i \in E_{n}} \lambda_{i}^{(n)} \mu_{i}^{(n)}<1+\eta
$$

Then player (I), pick signs $\left(\varepsilon_{i}^{(n)}\right)_{i \in E_{n}}$ so that if $x_{n}^{*}=\sum_{i \in E_{n}} \mu_{i}^{(n)} \varepsilon_{i}^{(n)} e_{i}^{*}$ then

$$
\left|x_{n}^{*}\left(T\left(\sum_{i \in E_{n}} \varepsilon_{i}^{(n)} \lambda_{i}^{(n)} e_{i}\right)\right)\right|>(1-\eta) \delta .
$$

That this is possible follows using the following probabilistic argument:

Let $r=\left(r_{j}\right)_{j \in E_{n}}$ be a Rademacher sequence, i.e. $r_{j}, j \in E_{n}$, are independent random variables on some probability space $(\Omega, \Sigma, \mathbb{P})$, with $\mathbb{P}\left(r_{j}=1\right)=\mathbb{P}\left(r_{j}=\right.$ $-1)=\frac{1}{2}$.

$$
\begin{aligned}
\mathbb{E}\left(\left(\sum_{i \in E_{n}} r_{i} \mu_{i}^{(n)} e_{i}^{*}\right)\left(T\left(\sum_{j \in E_{n}} r_{j} \lambda_{j}^{(n)} e_{j}\right)\right)\right) & =\mathbb{E}\left(\sum_{i, j \in E_{n}} r_{i} r_{j} \mu_{i}^{(n)} \lambda_{j}^{(n)} e_{j}^{*}\left(T\left(e_{i}\right)\right)\right) \\
& =\sum_{i \in E_{n}} \mu_{i}^{(n)} \lambda_{i}^{(n)} e_{i}^{*}\left(T\left(e_{i}\right)\right)>\delta(1-\eta) .
\end{aligned}
$$

It follows therefore that we can choose $\left(\varepsilon_{j}^{(n)}\right)_{j \in E_{n}}$ appropriately to satisfy (8).

After the game is completed, put

$$
x_{n}=\sum_{i \in E_{n}} \varepsilon_{i}^{(n)} \lambda_{i}^{(n)} e_{i} \quad \text { and } \quad x_{n}^{*}=\sum_{i \in E_{n}} \varepsilon_{i}^{(n)} \mu_{i}^{(n)} e_{i}^{*} .
$$

Conditions (i) to (iv) of Definition 3.4 are satisfied. Then (8) can be rewritten as

$$
\left|x_{n}^{*}\left(T\left(x_{n}\right)\right)\right| \geq(1-\eta) \delta .
$$

Furthermore, observe that for any $k<n$ we have

$$
\begin{aligned}
\left|x_{k}^{*}\left(T\left(x_{n}\right)\right)\right| & =\left|T^{*}\left(x_{k}^{*}\right)\left(x_{n}\right)\right| \leq\left\|T^{*}\left(x_{k}^{*}\right)\right\| \cdot \operatorname{dist}\left(x_{n}, W_{n}\right) \\
& \leq\|T\| \sqrt{C+\eta} \cdot \operatorname{dist}\left(x_{n}, W_{n}\right) \\
\left|x_{n}^{*}\left(T\left(x_{k}\right)\right)\right| & \leq\left\|T\left(x_{k}\right)\right\| \cdot \operatorname{dist}\left(x_{n}^{*}, G_{n}\right) \leq\|T\| \sqrt{C+\eta} \cdot \operatorname{dist}\left(x_{n}^{*}, G_{n}\right) .
\end{aligned}
$$

We conclude that $\sum_{n} \sum_{m \neq n}\left|x_{n}^{*}\left(T\left(x_{m}\right)\right)\right|<\eta$. A similar argument yields that $\left(x_{n}^{*}\right)_{n}$ is summably close to a block sequence of $\left(e_{i}^{*}\right)_{i}$. By Lemma 3.14 the diagonal operator $D: X \rightarrow X$ given by $D e_{n}=x_{n}^{*}\left(T\left(x_{n}\right)\right)$ is bounded. By assumption, for any $\xi>0$, the identity $(K(\delta-\eta)+\xi)$ factors through $D$ and if $\eta$ is sufficiently small then by the second part of Lemma 3.14 the identity $\left(\frac{(\lambda K(\delta-\eta)+\xi)(C+\eta)^{2}}{1-2 \lambda(K(\delta-\eta)+\xi) \eta}\right)$-factors through $T$. Recall that by (7) the function $K:(0, \infty) \rightarrow \mathbb{R}$ is continuous. As we could have picked $\eta$ and $\xi$ arbitrarily close to zero we deduce that the identity almost $\lambda K(\delta) C^{2}$-factors through $T$. 


\section{A Basic overview: multi-Parameter Lebesgue and Hardy spaces}

Here we give a preparation for the following sections in which we exhibit examples of strategically reproducible bases.

4.1. The multi-parameter Haar system. We denote by $\mathcal{D}$ the collection of all dyadic intervals in $[0,1)$, namely

$$
\mathcal{D}=\left\{\left[\frac{i-1}{2^{j}}, \frac{i}{2^{j}}\right): j \in \mathbb{N} \cup\{0\}, 1 \leq i \leq 2^{j}\right\} .
$$

For each $n \in \mathbb{N} \cup\{0\}$ we define $\mathcal{D}_{n}=\left\{I \in \mathcal{D}:|I|=2^{-n}\right\}$ and $\mathcal{D}^{n}=\cup_{k=0}^{n} \mathcal{D}_{k}$. We define the bijective function $\mathcal{O}: \mathcal{D} \rightarrow \mathbb{N}$ by

$$
\left[\frac{i-1}{2^{j}}, \frac{i}{2^{j}}\right) \mapsto 2^{j}+i-1 .
$$

The function $\mathcal{O}$ defines a linear order on $\mathcal{D}$. Recall that Haar system $\left(h_{I}\right)_{I \in \mathcal{D}}$ is defined as follows: if $I=\left[(i-1) / 2^{j}, i / 2^{j}\right)$ then set $I^{+}=\left[(i-1) / 2^{j},(2 i-1) / 2^{j+1}\right)$, $I^{-}=\left[(2 i-1) / 2^{j+1}, i / 2^{j}\right)$, and

$$
h_{I}=\chi_{I^{+}}-\chi_{I^{-}} .
$$

The $d$-parameter dyadic rectangles $\mathcal{R}_{d}$ are given by

$$
\mathcal{R}_{d}=\left\{I_{1} \times \cdots \times I_{d}: I_{1}, \ldots, I_{d} \in \mathcal{D}\right\},
$$

and the $d$-parameter tensor product Haar system $\left(h_{\bar{I}}\right)_{\bar{I} \in \mathcal{R}_{d}}$ is given by

$$
h_{\bar{I}}\left(t_{1}, t_{2}, \ldots t_{d}\right)=h_{I_{1}}\left(t_{1}\right) \cdot h_{I_{2}}\left(t_{2}\right) \cdot \ldots \cdot h_{I_{d}}\left(t_{d}\right), \quad t_{1}, t_{2}, \ldots t_{d} \in[0,1) .
$$

4.2. A linear order on $\mathcal{R}_{2}$. First, we define the bijective function $\mathcal{O}_{\mathbb{N}_{0}^{2}}: \mathbb{N}_{0}^{2} \rightarrow \mathbb{N}_{0}$ by

$$
\mathcal{O}_{\mathbb{N}_{0}^{2}}(m, n)= \begin{cases}n^{2}+m, & \text { if } m<n, \\ m^{2}+m+n, & \text { if } m \geq n .\end{cases}
$$

To see that $\mathcal{O}_{\mathbb{N}_{0}^{2}}$ is bijective consider that for each $k \in \mathbb{N}$ :

- $\mathcal{O}_{\mathbb{N}_{0}^{2}}(0,0)=0$,

- $m \mapsto \mathcal{O}_{\mathbb{N}_{0}^{2}}(m, k)$ maps $\{0, \ldots, k-1\}$ bijectively onto $\left\{k^{2}, \ldots, k^{2}+k-1\right\}$ and preserves the natural order on $\mathbb{N}_{0}$,

- $\mathcal{O}_{\mathbb{N}_{0}^{2}}(k, 0)=\mathcal{O}_{\mathbb{N}_{0}^{2}}(k-1, k)+1$,

- $n \mapsto \mathcal{O}_{\mathbb{N}_{0}^{2}}(k, n)$ maps $\{0, \ldots, k\}$ bijectively onto $\left\{k^{2}+k, \ldots, k^{2}+2 k\right\}$ and preserves the natural order on $\mathbb{N}_{0}$,

- $\mathcal{O}_{\mathbb{N}_{0}^{2}}(0, k+1)=\mathcal{O}_{\mathbb{N}_{0}^{2}}(k, k)+1$.

Now, let $<_{\ell}$ denote the lexicographic order on $\mathbb{R}^{3}$. For two dyadic rectangles $I_{k} \times J_{k} \in \mathcal{R}_{2}$ with $\left|I_{k}\right|=2^{-m_{k}},\left|J_{k}\right|=2^{-n_{k}}, k=0,1$, we define $I_{0} \times J_{0} \triangleleft I_{1} \times J_{1}$ if and only if

$$
\left(\mathcal{O}_{\mathbb{N}_{0}^{2}}\left(m_{0}, n_{0}\right), \inf I_{0}, \inf J_{0}\right)<_{\ell}\left(\mathcal{O}_{\mathbb{N}_{0}^{2}}\left(m_{1}, n_{1}\right), \inf I_{1}, \inf J_{1}\right) .
$$

Associated to the linear ordering $\triangleleft$ is the bijective index function $\mathcal{O}_{\triangleleft}: \mathcal{R}_{2} \rightarrow \mathbb{N}_{0}$ defined by

$$
\mathcal{O}_{\triangleleft}\left(R_{0}\right)<\mathcal{O}_{\triangleleft}\left(R_{1}\right) \Leftrightarrow R_{0} \triangleleft R_{1}, \quad R_{0}, R_{1} \in \mathcal{R}_{2} .
$$

See Figure 1 for a picture of $\mathcal{O}_{\triangleleft}$. 


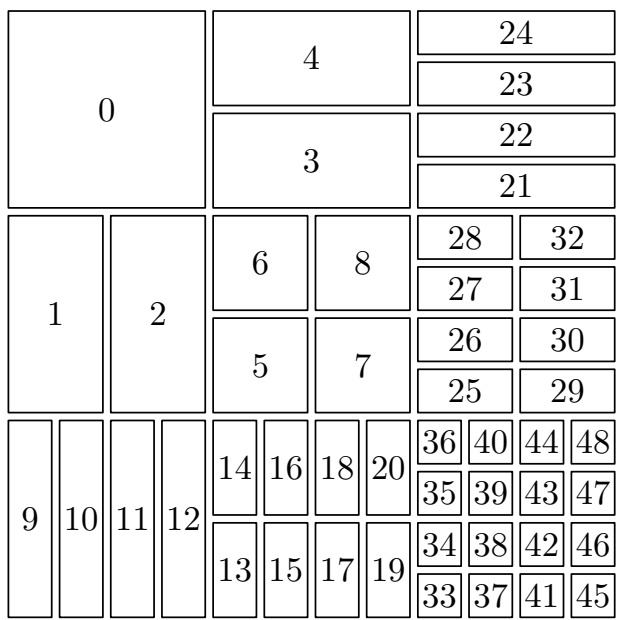

FiguRE 1. The first 49 rectangles and their indices $\mathcal{O}_{\triangleleft}$.

4.3. Multi-parameter Lebesgue spaces. Given $1 \leq p \leq \infty$, we define $L_{0}^{p}$ as the closed subspace of $L^{p}[0,1]$ given by

$$
L_{0}^{p}=\left\{f \in L^{p}: \int_{0}^{1} f(t) d t=0\right\} .
$$

Note that the Haar system $\left(h_{I}\right)_{I \in \mathcal{D}}$ ordered by $\mathcal{O}$ is a monotone basis for $L_{0}^{p}$, whenever $1 \leq p<\infty$. Moreover, $\left(h_{I}\right)_{I \in \mathcal{D}}$ is unconditional in $L_{0}^{p}$, whenever $1<$ $p<\infty$. The only reason for considering $L_{0}^{p}$ rather than $L^{p}$, is a notational one. Otherwise we would have to consider the first basis element of $L^{p}$, namely $\chi_{[0,1]}$, always separately from the other ones.

Given $\bar{p}=\left(p_{1}, \ldots, p_{d}\right)$, where $1 \leq p_{1}, \ldots, p_{d} \leq \infty$ we define the mixed norm Lebesgue space $L^{\bar{p}}$ by

$$
L^{\bar{p}}=L^{p_{1}}\left(L^{p_{2}}\left(L^{p_{3}}\left(\ldots\left(L^{p_{d}}\right)\right) \ldots\right)\right) .
$$

Moreover, we define the closed subspace $L_{0}^{\bar{p}}$ of $L^{\bar{p}}$ by

$$
L_{0}^{\bar{p}}=\left\{f \in L^{\bar{p}}: \int_{0}^{1} f\left(t_{1} \ldots, t_{d}\right) d t_{j}=0 \text { for all } 1 \leq j \leq d\right\} .
$$

The $d$-parameter tensor product Haar system $\left(h_{\bar{I}}\right)_{\bar{I} \in \mathcal{R}_{d}}$ is an unconditional basis for $L_{0}^{\bar{p}}$, whenever $\bar{p}=\left(p_{1}, \ldots, p_{d}\right)$ and $1<p_{1}, \ldots, p_{d}<\infty$. The dual of $L_{0}^{\bar{p}}$ is then $L_{0}^{\bar{q}}$, where $\bar{q}=\left(q_{1}, \ldots, q_{d}\right)$, and $\frac{1}{p_{i}}+\frac{1}{q_{i}}=1$, for $i=1,2, \ldots, d$.

In the following, we prove the equivalence of the $L^{\bar{p}}$ norm and the d-parametric square function norm in the reflexive case, i.e. $p=\left(p_{1}, \ldots, p_{d}\right)$ with $1<p_{1}, \ldots, p_{d}<$ $\infty$. The content of Proposition 4.1 was known and used by Capon 4; for the convenience of the reader, we provide a detailed exposition, below. 
Proposition 4.1. Let $d \in \mathbb{N}, 1<p_{1}, p_{2}, \ldots, p_{d}<\infty$ and $\bar{p}=\left(p_{1}, p_{2}, \ldots, p_{d}\right)$. For $f=\sum_{\bar{I} \in \mathcal{R}_{d}} a_{\bar{I}} h_{\bar{I}} \in L_{0}^{\bar{p}}$ we define

$$
\|f\|_{\bar{p}}=\left(\int_{0}^{1}\left(\ldots \int_{0}^{1}\left(\int_{0}^{1}\left(\sum_{\bar{I} \in \mathcal{R}_{d}} a_{\bar{I}}^{2} h_{\bar{I}}^{2}\left(t_{1}, \ldots t_{d}\right)\right)^{\frac{p_{d}}{2}} d t_{d}\right)^{\frac{p_{d-1}}{p_{d}}} \ldots\right)^{\frac{p_{1}}{p_{2}}} d t_{1}\right)^{\frac{1}{p_{1}}} .
$$

Then $\|\cdot\|_{\bar{p}}$ is an equivalent norm on $L_{0}^{\bar{p}}$. The dual norm to $\|\cdot\|_{\bar{p}}$ is equivalent (with constants depending on $\bar{p})$ to $\|\cdot\|_{\bar{q}}$, where $\bar{q}=\left(q_{1}, q_{2}, \ldots q_{d}\right)$, and $\frac{1}{p_{i}}+\frac{1}{q_{i}}=1$, for $i=1,2, \ldots, d$.

Proof. We show 10 by induction on $d$. By the unconditionality of the Haar system in $L^{p}, 1<p<\infty$ (theorem of Paley-Marcinkiewicz [23] and [14]) the statement is true for $d=1$. Assume that we proved (10) for $L_{0}^{\left(p_{2}, \ldots, p_{d}\right)}$. In the following, we use the abbreviations $X_{j}=L_{0}^{\left(p_{1}, \ldots, p_{j}\right)}$ and $Y_{j}=L_{0}^{\left(p_{j}, \ldots, p_{d}\right)}\left(\ell^{2}\left(\mathcal{R}_{d-1}\right)\right), 1 \leq j \leq d$. Since $L_{0}^{\left(p_{2}, \ldots, p_{d}\right)}$ has the UMD property 16 , page II.12] we obtain that

$$
\|f\|_{L_{0}^{\bar{p}}} \sim_{\bar{p}}\left(\int_{0}^{1}\left\|\sum_{\bar{I}} \varepsilon_{I_{1}} a_{\bar{I}} h_{I_{1}}\left(t_{1}\right) h_{I_{2}, \ldots, I_{d}}\right\|_{\left(p_{2}, \ldots, p_{d}\right)}^{p_{1}} d t_{1}\right)^{\frac{1}{p_{1}}}
$$

for all choices of signs $\varepsilon_{I_{1}}, I_{1} \in \mathcal{D}$. Hence, by averaging and Kahane's inequality 12 , Theorem 4], we obtain

$$
\begin{aligned}
\|f\|_{L_{0}^{\bar{p}}} & \sim \int_{\bar{p}}\left(\int_{0}^{1}\left(\mathbb{E}_{\varepsilon}\left\|\sum_{\bar{I}} \varepsilon_{I_{1}} a_{\bar{I}} h_{I_{1}}\left(t_{1}\right) h_{I_{2}, \ldots, I_{d}}\right\|_{\left(p_{2}, \ldots, p_{d}\right)}\right)^{p_{1}} d t_{1}\right)^{\frac{1}{p_{1}}} \\
& =\left\|\mathbb{E}_{\varepsilon}\right\| \sum_{\bar{I}} \varepsilon_{I_{1}} a_{\bar{I}} h_{I_{1}} h_{I_{2}, \ldots, I_{d}}\left\|_{\left(p_{2}, \ldots, p_{d}\right)}\right\|_{X_{1}} .
\end{aligned}
$$

By induction hypothesis, we obtain

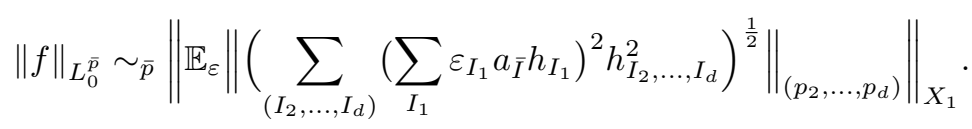

Now, observe that

$$
\begin{aligned}
\mathbb{E}_{\varepsilon} \| & \left(\sum_{\left(I_{2}, \ldots, I_{d}\right)}\left(\sum_{I_{1}} \varepsilon_{I_{1}} a_{\bar{I}} h_{I_{1}}\right)^{2} h_{I_{2}, \ldots, I_{d}}^{2}\right)^{\frac{1}{2}} \|_{\left(p_{2}, \ldots, p_{d}\right)} \\
& =\mathbb{E}_{\varepsilon}\|\| \sum_{I_{1}} \varepsilon_{I_{1}}\left(a_{\bar{I}} h_{I_{1}} h_{I_{2}, \ldots, I_{d}}\right)_{\left(I_{2}, \ldots, I_{d}\right)}\left\|_{\ell^{2}\left(I_{2}, \ldots, I_{d}\right)}\right\|_{\left(p_{2}, \ldots, p_{d}\right)} \\
& =\mathbb{E}_{\varepsilon}\left\|\sum_{I_{1}} \varepsilon_{I_{1}}\left(a_{\bar{I}} h_{I_{1}} h_{I_{2}, \ldots, I_{d}}\right)_{\left(I_{2}, \ldots, I_{d}\right)}\right\|_{Y_{2}} .
\end{aligned}
$$

Kahane's inequality yields

$$
\begin{aligned}
\mathbb{E}_{\varepsilon} \| & \sum_{I_{1}} \varepsilon_{I_{1}}\left(a_{\bar{I}} h_{I_{1}} h_{I_{2}, \ldots, I_{d}}\right)_{\left(I_{2}, \ldots, I_{d}\right)} \|_{Y_{2}} \\
& \sim_{\bar{p}}\left(\mathbb{E}_{\varepsilon}\left\|\sum_{I_{1}} \varepsilon_{I_{1}}\left(a_{\bar{I}} h_{I_{1}} h_{I_{2}, \ldots, I_{d}}\right)_{\left(I_{2}, \ldots, I_{d}\right)}\right\|_{Y_{2}}^{p_{2}}\right)^{\frac{1}{p_{2}}}
\end{aligned}
$$




$$
=\left(\int \mathbb{E}_{\varepsilon}\left\|\sum_{I_{1}} \varepsilon_{I_{1}}\left(a_{\bar{I}} h_{I_{1}} h_{I_{2}, \ldots, I_{d}}\right)_{\left(I_{2}, \ldots, I_{d}\right)}\right\|_{Y_{3}} d t_{2}\right)^{\frac{1}{p_{2}}}
$$

Combining our estimates yields

$$
\begin{aligned}
\|f\|_{L_{0}^{\bar{p}}} & \sim_{\bar{p}}\left\|\left(\int \mathbb{E}_{\varepsilon}\left\|\sum_{I_{1}} \varepsilon_{I_{1}}\left(a_{\bar{I}} h_{I_{1}} h_{I_{2}, \ldots, I_{d}}\right)_{\left(I_{2}, \ldots, I_{d}\right)}\right\|_{Y_{3}} d t_{2}\right)^{\frac{1}{p_{2}}}\right\|_{X_{1}} \\
& =\left\|\mathbb{E}_{\varepsilon}\right\| \sum_{I_{1}} \varepsilon_{I_{1}}\left(a_{\bar{I}} h_{I_{1}} h_{I_{2}, \ldots, I_{d}}\right)_{\left(I_{2}, \ldots, I_{d}\right)}\left\|_{Y_{3}}\right\|_{X_{2}} .
\end{aligned}
$$

With the same argument, we obtain

$$
\|f\|_{L_{0}^{\bar{p}} \sim} \sim_{\bar{p}}\left\|\mathbb{E}_{\varepsilon}\right\| \sum_{I_{1}} \varepsilon_{I_{1}}\left(a_{\bar{I}} h_{I_{1}} h_{I_{2}, \ldots, I_{d}}\right)_{\left(I_{2}, \ldots, I_{d}\right)}\left\|_{Y_{4}}\right\|_{X_{3}}
$$

Continuing in this fashion yields

$$
\|f\|_{L_{0}^{\bar{p}} \sim_{\bar{p}}}\left\|\mathbb{E}_{\varepsilon}\right\| \sum_{I_{1}} \varepsilon_{I_{1}}\left(a_{\bar{I}} h_{I_{1}} h_{I_{2}, \ldots, I_{d}}\right)_{\left(I_{2}, \ldots, I_{d}\right)}\left\|_{\ell^{2}\left(\mathcal{R}_{d-1}\right)}\right\|_{L_{0}^{\bar{p}}} \cdot
$$

Applying Kahane's inequality one last time, we obtain

$$
\begin{aligned}
\mathbb{E}_{\varepsilon} \| & \sum_{I_{1}} \varepsilon_{I_{1}}\left(a_{\bar{I}} h_{I_{1}} h_{I_{2}, \ldots, I_{d}}\right)_{\left(I_{2}, \ldots, I_{d}\right)} \|_{\ell^{2}\left(\mathcal{R}_{d-1}\right)} \\
& \sim_{\bar{p}}\left(\mathbb{E}_{\varepsilon}\left\|\left(\sum_{I_{1}} \varepsilon_{I_{1}} a_{\bar{I}} h_{I_{1}} h_{I_{2}, \ldots, I_{d}}\right)_{\left(I_{2}, \ldots, I_{d}\right)}\right\|_{\ell^{2}\left(\mathcal{R}_{d-1}\right)}^{2}\right)^{1 / 2} .
\end{aligned}
$$

Note that the last expression is equal to

$$
\begin{aligned}
\left(\sum_{I_{2}, \ldots, I_{d}} \mathbb{E}_{\varepsilon}\left|\sum_{I_{1}} \varepsilon_{I_{1}} a_{\bar{I}} h_{I_{1}} h_{I_{2}, \ldots, I_{d}}\right|^{2}\right)^{1 / 2} & =\left(\sum_{I_{2}, \ldots, I_{d}} \sum_{I_{1}} a_{\bar{I}}^{2} h_{I_{1}}^{2} h_{I_{2}, \ldots, I_{d}}^{2}\right)^{1 / 2} \\
& =\left(\sum_{\bar{I}} a_{\bar{I}}^{2} h_{\bar{I}}^{2}\right)^{1 / 2} .
\end{aligned}
$$

Combining (11) with 12 and 13 yields 10$)$.

4.4. Hardy spaces. We define the dyadic Hardy spaces $H^{p}, 1 \leq p<\infty$ as the completion of

$$
\operatorname{span}\left\{h_{I}: I \in \mathcal{D}\right\}
$$

under the square function norm

$$
\left\|\sum_{I \in \mathcal{D}} a_{I} h_{I}\right\|_{H^{p}}=\left\|\mathbb{S}\left(\sum_{I \in \mathcal{D}} a_{I} h_{I}\right)\right\|_{L^{p}},
$$

where the square function $\mathbb{S}$ is given by

$$
\mathbb{S}\left(\sum_{I \in \mathcal{D}} a_{I} h_{I}\right)=\left(\sum_{I \in \mathcal{D}} a_{I}^{2} h_{I}^{2}\right)^{1 / 2}
$$

for all scalar sequences $\left(a_{I}\right)_{I \in \mathcal{D}}$.

The bi-parameter dyadic Hardy spaces $H^{p}\left(H^{q}\right), 1 \leq p, q<\infty$ are defined as the completion of

$$
\operatorname{span}\left\{h_{\bar{I}}: \bar{I} \in \mathcal{R}_{2}\right\}
$$


under the bi-parameter square function norm

$$
\left\|\sum_{\bar{I} \in \mathcal{R}_{2}} a_{\bar{I}} h_{\bar{I}}\right\|_{H^{p}\left(H^{q}\right)}=\left\|\mathbb{S}\left(\sum_{\bar{I} \in \mathcal{R}_{2}} a_{\bar{I}} h_{\bar{I}}\right)\right\|_{L^{p}\left(L^{q}\right)},
$$

where the bi-parameter square function $\mathbb{S}$ is given by

$$
\mathbb{S}\left(\sum_{\bar{I} \in \mathcal{R}_{2}} a_{\bar{I}} h_{\bar{I}}\right)=\left(\sum_{\bar{I} \in \mathcal{R}_{2}} a_{\bar{I}}^{2} h_{\bar{I}}^{2}\right)^{1 / 2}
$$

for all scalar sequences $\left(a_{\bar{I}}\right)_{\bar{I} \in \mathcal{R}_{2}}$.

The following Lemma is taken from 13].

Lemma 4.2. For $m \in \mathbb{N}$, let $\mathcal{X}_{m}$ and $\mathcal{Y}_{m}$ be non-empty, finite families of pairwise disjoint dyadic intervals, define $f_{m}=\sum_{I \in \mathcal{X}_{m}, J \in \mathcal{Y}_{m}} h_{I \times J}$, and let $1 \leq p, q<\infty$. Suppose in addition that:

- $\mathcal{X}_{m} \cap \mathcal{X}_{n}=\emptyset$ or $\mathcal{Y}_{m} \cap \mathcal{Y}_{n}=\emptyset$ whenever $m, n \in \mathbb{N}$ are distinct;

- $\bigcup \mathcal{X}_{m}=\bigcup \mathcal{X}_{n}$ and $\bigcup \mathcal{Y}_{m}=\bigcup \mathcal{Y}_{n}$ for all $m, n \in \mathbb{N}$.

Then for each $\gamma \in \ell^{\infty}(\mathcal{R})$ with $\|\gamma\|_{\infty} \leq 1$, the operator $M_{\gamma}$ defined as the linear extension of the map $h_{I \times J} \mapsto \gamma_{I \times J} h_{I \times J}$ is bounded by 1, both as a map from $H^{p}\left(H^{q}\right)$ to itself and from $H^{p}\left(H^{q}\right)^{*}$ to itself. Moreover,

(i) for each $g \in H^{p}\left(H^{q}\right)^{*}$, $\sup _{\gamma \in B_{\ell}(\mathcal{R})}\left|\left\langle M_{\gamma} f_{m}, g\right\rangle\right| \rightarrow 0$ as $m \rightarrow \infty$;

(ii) for each $g \in H^{p}\left(H^{q}\right) \sup _{\gamma \in B_{\ell}(\mathcal{R})}\left|\left\langle M_{\gamma} g, f_{m}\right\rangle\right| \rightarrow 0$ as $m \rightarrow \infty$.

4.5. Collections of dyadic intervals. We introduce convenient notation and gather basic facts of collections of dyadic intervals.

Notation 4.3.

(i) For $\mathcal{A} \subset \mathcal{D}$ set $\mathscr{G}_{0}(\mathcal{A})=\{I \in \mathcal{A}: I$ is maximal with respect to inclusion $\}$.

(ii) For $\mathcal{A} \subset \mathcal{D}$ recursively define for $n \in \mathbb{N}$ the collection

$$
\mathscr{G}_{n}(\mathcal{A})=\mathscr{G}_{0}\left(\mathcal{A} \backslash\left(\cup_{k=0}^{n-1} \mathscr{G}_{k}(\mathcal{A})\right)\right) .
$$

For every $n \in \mathbb{N}$ and $I \in \mathscr{G}_{n+1}(\mathcal{A})$ there is a unique $J \in \mathscr{G}_{n}(\mathcal{A})$ with $I \subset J$. In paricular, $\mathscr{G}_{n+1}(\mathcal{A})^{*} \subset \mathscr{G}_{n}(\mathcal{A})^{*}$

(iii) For $\mathcal{A} \subset \mathcal{D}$ set $\lim \sup \mathcal{A}=\cap_{n} \mathscr{G}_{n}(\mathcal{A})^{*}$.

(iv) For a finite $\mathcal{H} \subset \mathcal{D}$, consisting of pairwise disjoint intervals, and $\bar{\varepsilon}=$ $(\varepsilon)_{I \in \mathcal{H}} \in\{-1,1\}^{\mathcal{H}}$ we define

$$
\mathcal{H}_{\bar{\varepsilon}}^{*}=\left[\sum_{I \in \mathcal{H}} \varepsilon_{I} h_{I}=1\right] \text { and } \mathcal{H}_{-\bar{\varepsilon}}^{*}=\left[\sum_{I \in \mathcal{H}} \varepsilon_{I} h_{I}=-1\right] .
$$

These two sets have measure $\left|\mathcal{H}^{*}\right| / 2$ and they form a partition of $\mathcal{H}^{*}$.

(v) For $\mathcal{A} \subset \mathcal{D}, n, k \in \mathbb{N}$, with $k \geq n$ and a finite $\mathcal{H} \subset \mathscr{G}_{n}(\mathcal{A})$ define the collection

$$
\mathcal{H}_{k}^{\text {succ }}=\left\{I \in \mathscr{G}_{k}(\mathcal{A}): I \subset J \text { for some } J \in \mathcal{H}\right\} .
$$

For any $\bar{\varepsilon} \in\{-1,1\}^{\mathcal{H}}$ define the sets

$\mathcal{H}_{\bar{\varepsilon}, k}^{\text {succ }}=\left\{I \in \mathscr{G}_{k}(\mathcal{A}): I \subset \mathcal{H}_{\bar{\varepsilon}}^{*}\right\}$ and $\mathcal{H}_{-\bar{\varepsilon}, k}^{\text {succ }}=\left\{I \in \mathscr{G}_{k}(\mathcal{A}): I \subset \mathcal{H}_{-\bar{\varepsilon}}^{*}\right\}$.

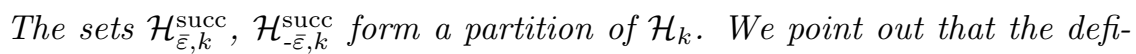
nitions of $\mathcal{H}_{k}^{\text {succ }}, \mathcal{H}_{\bar{\varepsilon}, k}^{\text {succ }}$, and $\mathcal{H}_{-\bar{\varepsilon}, k}^{\text {succ }}$ depend on the ambient collection $\mathcal{A}$.

Lemma 4.4 and 4.5 will be used in Section 6 . 
Lemma 4.4. Let $\mathcal{A} \subset \mathcal{D}$. Then for any $\kappa>0$ there is $\tilde{\mathcal{A}} \subset \mathcal{A}$ so that for each $k \in \mathbb{N}$ we have

(i) $\mathscr{G}_{k}(\tilde{\mathcal{A}})$ is finite and $\mathscr{G}_{k}(\tilde{\mathcal{A}}) \subset \mathscr{G}_{k}(\mathcal{A})$ and

(ii) $|\lim \sup (\tilde{\mathcal{A}})| \geq|\lim \sup (\mathcal{A})|-\kappa$.

Proof. Pick a finite $\mathcal{B}_{0} \subset \mathscr{G}_{0}(\mathcal{A})$ with $\left|\mathscr{G}_{0}(\mathcal{A})^{*} \backslash \mathcal{B}_{0}^{*}\right|<\kappa / 2$. Recursively for $k \in \mathbb{N}$, if $\mathcal{C}=\mathcal{B}_{k-1}$, pick $\mathcal{B}_{k} \subset \mathcal{C}_{k}^{\text {succ }}$ with $\left|\left(\mathcal{C}_{k}^{\text {succ }}\right)^{*} \backslash \mathcal{B}_{k}^{*}\right|<\kappa / 2^{k+1}$. Set $\tilde{\mathcal{A}}=\cup_{k=0}^{\infty} \mathcal{B}_{k}$. One can check by induction that for all $k \in \mathbb{N}$ we have $\mathscr{G}_{k}(\tilde{\mathcal{A}})=\mathcal{B}_{k} \subset \mathscr{G}_{k}(\mathcal{A})$ and that $\left|\mathscr{G}_{k}(\mathcal{A})^{*} \backslash \mathcal{B}_{k}^{*}\right| \leq \sum_{i=1}^{k} \kappa / 2^{i}$. The conclusion easily follows.

Lemma 4.5. Let $\mathcal{A} \subset \mathcal{D}, n \in \mathbb{N}, \kappa \in(0,1 / 2)$, and $\mathcal{H} \subset \mathscr{G}_{n}(\mathcal{A})$ be a non-empty finite collection so that if $A=\lim \sup \mathcal{A}$ then $\left|\mathcal{H}^{*} \cap A\right|>(1-\kappa)\left|\mathcal{H}^{*}\right|$. The following hold.

(i) For any $\bar{\varepsilon} \in\{-1,1\}^{\mathcal{H}}$, if $C=\mathcal{H}_{\bar{\varepsilon}}^{*}$ or $C=\mathcal{H}_{-\bar{\varepsilon}}^{*}$ we have

$$
\frac{\left|\mathcal{H}^{*}\right|}{2} \geq|C \cap A|>(1-2 \kappa)|C|=(1-2 \kappa) \frac{\left|\mathcal{H}^{*}\right|}{2} .
$$

(ii) For any $\delta>0$ there exists $k_{0} \in \mathbb{N}$ so that for all $k \geq k_{0}$ and $\bar{\varepsilon} \in\{-1,1\}^{\mathcal{H}}$, if $C=\left(\mathcal{H}_{\bar{\varepsilon}, k}^{\text {succ }}\right)^{*}$ or $C=\left(\mathcal{H}_{-\bar{\varepsilon}, k}^{\text {succ }}\right)^{*}$ we have $|C \cap A| \geq(1-\delta)|C|$.

Proof. For the proof of (i) let $C=\mathcal{H}_{\bar{\varepsilon}}^{*}$. Recall that $\left|\mathcal{H}_{\bar{\varepsilon}}^{*}\right|=\left|\mathcal{H}_{-\bar{\varepsilon}}^{*}\right|=\left|\mathcal{H}^{*}\right| / 2$. Now,

$$
\begin{aligned}
(1-\kappa) 2\left|\mathcal{H}_{\bar{\varepsilon}}^{*}\right| & =(1-\kappa)|| \mathcal{H}^{*}|<| \mathcal{H}^{*} \cap A|=| \mathcal{H}_{\bar{\varepsilon}}^{*} \cap A|+| \mathcal{H}_{-\bar{\varepsilon}}^{*} \cap A \mid \\
& \leq\left|\mathcal{H}_{\bar{\varepsilon}}^{*} \cap A\right|+\left|\mathcal{H}_{-\bar{\varepsilon}}^{*}\right|=\left|\mathcal{H}_{\bar{\varepsilon}}^{*} \cap A\right|+\left|\mathcal{H}_{\bar{\varepsilon}}^{*}\right|
\end{aligned}
$$

which yields $\left|\mathcal{H}_{\bar{\varepsilon}}^{*} \cap A\right|>(1-2 \kappa)\left|\mathcal{H}_{\bar{\varepsilon}}^{*}\right|$. The same argument works for $C=\mathcal{H}_{-\bar{\varepsilon}}$.

We now prove (ii). As there are finitely many choices of $\bar{\varepsilon} \in\{-1,1\}^{\mathcal{H}}$ it suffices to prove it by fixing one of them. Observe the sequence $\left(\left(\mathcal{H}_{\bar{\varepsilon}, k}^{\text {succ }}\right)^{*}\right)_{k}$ is decreasing so we can define $\left(\mathcal{H}_{\bar{\varepsilon}, \infty}^{\text {succ }}\right)^{*}=\cap_{k}\left(\mathcal{H}_{\bar{\varepsilon}, k}^{\text {succ }}\right)^{*}=\mathcal{H}_{\bar{\varepsilon}}^{*} \cap A=\cap_{k}\left(\left(\mathcal{H}_{\bar{\varepsilon}, k}^{\text {succ }}\right)^{*} \cap A\right)$. For one we obtain $\lim _{k}\left|\left(\mathcal{H}_{\bar{\varepsilon}, k}^{\text {succ }}\right)^{*}\right|=\left|\mathcal{H}_{\bar{\varepsilon}}^{*} \cap A\right| \geq(1-2 \kappa)\left|\mathcal{H}_{\bar{\varepsilon}}^{*} \cap A\right|>0$. Since also $\lim _{k}\left|\left(\mathcal{H}_{\bar{\varepsilon}, k}^{\text {succ }}\right)^{*} \cap A\right|=$ $\left|\mathcal{H}_{\bar{\varepsilon}}^{*} \cap A\right|$ we obtain $\lim _{k}\left(\left|\mathcal{H}_{\bar{\varepsilon}}^{*}\right| /\left|\mathcal{H}_{\bar{\varepsilon}}^{*} \cap A\right|\right)=1$ which yields the desired conclusion.

\section{Strategical Reproducibility of the HaAr system}

We establish that the Haar system is strategically reproducible in the following classical Banach spaces:

(i) The multi-parameter tensor product Haar system is strategically reproducible in the reflexive mixed norm Lebesgue spaces $L^{\left(p_{1}, \ldots, p_{d}\right)}, 1<p_{i}<\infty, 1 \leq i \leq d$, $d \in \mathbb{N}$, in the sense of Definition 3.1 .

(ii) The one-parameter Haar system in $H^{1}$ is strategically reproducible according to Definition 3.3 .

(iii) The two-parameter tensor product Haar system is strategically reproducible in the two-parameter mixed norm Hardy spaces $H^{p}\left(H^{q}\right), 1 \leq p, q<\infty$ in the sense of Definition 3.3 .

5.1. The Haar system in multi-parameter Lebesgue spaces. Here we show that $\left(h_{\bar{I}}\right)$ is strategically reproducible in $L^{\left(p_{1}, \ldots, p_{d}\right)}, 1<p_{i}<\infty, 1 \leq i \leq d, d \in \mathbb{N}$, in the sense of Definition 3.1. We exploit the fact that $\left(h_{\bar{I}}\right)$ is an unconditional basis for $L^{\left(p_{1}, \ldots, p_{d}\right)}$, and that $\left(L^{\left(p_{1}, \ldots, p_{d}\right)}\right)^{*}=L^{\left(q_{1}, \ldots, q_{d}\right)}$, where $\frac{1}{p_{i}}+\frac{1}{q_{i}}=1,1 \leq i \leq d$.

Theorem 5.1. Let $d \in \mathbb{N}, 1<p_{1}, p_{2}, \ldots, p_{d}<\infty$ and put $\bar{p}=\left(p_{1}, p_{2}, \ldots, p_{d}\right)$. Then $\left(h_{\bar{I}}\right)$ with an appropriate linear order is strategically reproducible in $L_{0}^{\bar{p}}$. 
Proof. We linearly order $\mathcal{R}_{d}$ into $\left(\bar{I}^{(k)}\right)=\left(I_{1}^{(k)}, I_{2}^{(k)}, \ldots, I_{d}^{(k)}\right)_{k=1}^{\infty}$ in a manner which is compatible with " $\subset$ ", i.e., we assume that if for $m, k \in \mathbb{N}$, we have $I_{i}^{(m)} \subset I_{i}^{(k)}$, for $i=1,2, \ldots, d$, then $m \geq k$. We also linearly order the Haar basis of $L_{0}^{\bar{p}}$ into $\left(h_{\bar{I}^{(k)}}\right)_{k=1}^{\infty}$. For any $\bar{I} \in \mathcal{R}_{d}, n(\bar{I})$ denotes the number $n \in \mathbb{N}$ so that $\bar{I}=\bar{I}^{(n)}$.

Then a winning strategy for player (II) will look as follows:

Assume he has chosen $b_{1}, b_{2}, \ldots b_{l}$, and $b_{1}^{*}, b_{2}^{*}, \ldots b_{l}^{*}$ in $L_{0}^{\bar{p}}$ and $\left(L_{0}^{\bar{p}}\right)^{*}$. Assume that $b_{j}, 1 \leq j \leq l$ is of the following form:

$$
b_{j}=\sum_{\substack{\bar{J}=\left(J_{1}, \ldots J_{d}\right) \in \mathcal{D}_{k(j, 1)} \times \mathcal{D}_{k(j, 2)} \times \ldots \times \mathcal{D}_{k(j, d)} \\ J_{1} \times J_{2} \times \ldots \times J_{d} \subset I_{1}^{(j)} \times I_{2}^{(j)} \times \ldots I_{d}^{(j)}}} h_{\bar{J}}
$$

with $k(j, i) \leq k\left(j^{\prime}, i\right)$, if $j \leq j^{\prime}$, and $k(j, i)<k\left(j^{\prime}, i\right)$ if $I_{i}^{(j)} \supsetneq I_{i}^{\left(j^{\prime}\right)}$, for $i=$ $1,2 \ldots d$. We also assume that for all $j=1,2 \ldots l$ we have $n(\bar{J})>n_{j}$ for all $\bar{J} \in \mathcal{D}_{k(j, 2)} \times \ldots \times \mathcal{D}_{k(j, d)}$, where $n_{j}$ was the $j$-th move of player (I) and, moreover, we assume that

$$
b_{j}^{*}=\left(\prod_{s=1}^{d}\left|I_{s}^{(j)}\right|\right)^{-1} b_{j} .
$$

Thus $\left(b_{j}^{*}\right)_{j=1}^{l}$ is biorthogonal to $\left(b_{j}\right)_{j=1}^{l}$ and $\left|b_{j}\right|=\left|h_{\bar{I}^{(j)}}\right|$, for $j=1,2 \ldots l$ which means that with respect to $\|\cdot\|_{\bar{p}}$ and, using 10 in Proposition 4.1. $\left(b_{j}\right)_{j=1}^{l}$ and $\left(b_{j}^{*}\right)_{j=1}^{l}$ are isometrically equivalent to $\left(h_{\bar{I}_{j}}\right)_{j=1}^{l}$ and $\left(h_{\bar{I}_{j}}^{*}\right)_{j=1}^{l}$, respectively.

Assuming now the $(l+1)$ st move of player (I) is $n_{l+1}$, player (II) can proceed as follows. For $j=1,2 \ldots d$, he chooses $k(l+1, j) \geq \max _{m \leq l} k(m, j)$ so that $k(l+1, j)>k(m, j)$ if $I_{j}^{(l+1)} \subsetneq I_{j}^{(m)}$ and so that for all $\bar{J} \in \prod_{j=1}^{d} \mathcal{D}_{k(l+1, j)}$, we have $n(\bar{J}) \geq n_{l+1}$.

5.2. The Haar system in $H^{1}$. Here, we use the Gamlen-Gaudet construction 10 (see also 18, 21]) to show that the one-parameter Haar system in $H^{1}$ is strategically reproducible according to Definition 3.3 .

For convenience, we introduce the following notation: We define $e_{I}=h_{I} /|I|$, $I \in \mathcal{D}$, thus $\left(e_{I}\right)_{I \in \mathcal{D}}$ forms a 1-unconditional normalized basis for $H^{1}$. Note that $e_{I}^{*}=h_{I} \in\left(H^{1}\right)^{*}, I \in \mathcal{D}$. Finally, we will identify a dyadic interval $I \in \mathcal{D}$ with $\mathcal{O}(I)$, e.g.

$$
\mathcal{E}_{k} \leftrightarrow \mathcal{E}_{I}, \quad x_{k} \leftrightarrow x_{I}, \quad x_{k}^{*} \leftrightarrow x_{I}^{*}, \quad I \in \mathcal{D}, \mathcal{O}(I)=k .
$$

Recall that the linear order $\mathcal{O}$ was introduced in Section 4.1 .

Theorem 5.2. The normalized Haar basis is strategically reproducible in $H^{1}$.

Proof. Here, we show that $\left(e_{I}\right)_{I \in \mathcal{D}}$ is $\sqrt{2}$-strategically reproducible in $H^{1}$.

We start the game with turn 1 . In step 1, player (I) chooses $\eta_{[0,1)}>0, W_{[0,1)} \in$ $\operatorname{cof}\left(H^{1}\right)$, and $G_{[0,1)} \in \operatorname{cof}_{w^{*}}\left(\left(H^{1}\right)^{*}\right)$. In step 2, player (II) selects one of the sets $\mathcal{E}_{[0,1)}^{(j)}=\mathcal{D}_{j}, j \in \mathbb{N}$. Put

$$
\begin{aligned}
& d_{[0,1)}^{(j)}=\sum_{K \in \mathcal{E}_{[0,1)}^{(j)}}|K| e_{K}, \\
& d_{[0,1)}^{*(j)}=\sum_{K \in \mathcal{E}_{[0,1)}^{(j)}} e_{K}^{*},
\end{aligned}
$$


and note that $\left(d_{[0,1)}^{(j)}\right)_{j=1}^{\infty}$ converges to 0 in the weak topology of $H^{1}$ and the sequence $\left(d_{[0,1)}^{*(j)}\right)_{j=1}^{\infty}$ converges in the $\mathrm{w}^{*}$ topology in $\left(H^{1}\right)^{*}$. Hence, there exists an index $j_{0}$ such that $\operatorname{dist}_{H^{1}}\left(d_{[0,1)}^{\left(j_{0}\right)}, W_{[0,1)}\right)<\eta_{[0,1)}$ and $\operatorname{dist}_{\left(H^{1}\right)^{*}}\left(d_{[0,1)}^{*\left(j_{0}\right)}, G_{[0,1)}\right)<\eta_{[0,1)}$. Player (II) concludes step 2 by choosing

$$
\mathcal{E}_{[0,1)}=\mathcal{E}_{[0,1)}^{\left(j_{0}\right)}
$$

and

$$
\lambda_{K}^{[0,1)}=|K| \quad \text { and } \quad \mu_{K}^{[0,1)}=1, \quad K \in \mathcal{E}_{[0,1)} .
$$

In step 3, player $(I)$ chooses $\left(\varepsilon_{K}^{([0,1))}\right)_{K \in \mathcal{E}_{[0,1)}} \in\{-1,1\}^{\mathcal{E}_{[0,1)}}$.

Assume that the game has already been played for $k=\mathcal{O}(I)-1$ turns. We will now play out turn $k+1=\mathcal{O}(I)$. In step 1 , player (I) chooses $\eta_{I}>0, W_{I} \in \operatorname{cof}\left(H^{1}\right)$, and $G_{I} \in \operatorname{cof}_{w^{*}}\left(\left(H^{1}\right)^{*}\right)$. In step 2, it is player II's choice to select the finite sets $\mathcal{E}_{I} \subset \mathcal{D}$. We will now describe this procedure. Note that since $W_{I} \in \operatorname{cof}\left(H^{1}\right)$ and $G_{I} \in \operatorname{cof}_{w^{*}}\left(\left(H^{1}\right)^{*}\right)$, there exist $f_{j} \in H^{1}, g_{j} \in\left(H^{1}\right)^{*}, 1 \leq j \leq N_{I}$, such that $W_{I}=\left\{g_{1}, \ldots, g_{N_{I}}\right\}_{\perp}$ and $G_{I}=\left\{f_{1}, \ldots, f_{N_{I}}\right\}^{\perp}$. Let $\widetilde{I}$ denote the dyadic predecessor of $I$, i.e. $\widetilde{I}$ is the unique dyadic interval that satisfies $\widetilde{I} \supsetneq I$ and $|\widetilde{I}|=2|I|$. Note that $\mathcal{O}(\widetilde{I}) \leq k$; specifically, $\mathcal{E}_{\widetilde{I}}$ has already been defined. Put

$$
X_{I}= \begin{cases}\left\{K^{\ell}: K \in \mathcal{E}_{\widetilde{I}}\right\}, & \text { if } I \text { is the left successor of } \widetilde{I}, \\ \left\{K^{r}: K \in \mathcal{E}_{\widetilde{I}}\right\}, & \text { if } I \text { is the right successor of } \widetilde{I},\end{cases}
$$

where $K^{\ell}$ denotes the left successor of $K$ and $K^{r}$ denotes the right successor of $K$. We note that by induction $\left|X_{I}\right|=|I|$.

$$
\begin{aligned}
d_{I}^{(j)} & =\sum_{\substack{K \in \mathcal{D}_{j} \\
K \subset X_{I}}}|K| e_{K}, \quad j \in \mathbb{N}, \\
d_{I}^{*(j)} & =\sum_{\substack{K \in \mathcal{D}_{j} \\
K \subset X_{I}}} e_{K}^{*}, \quad j \in \mathbb{N} .
\end{aligned}
$$

Since the sequence $\left(d_{I}^{(j)}\right)_{j=1}^{\infty}$ converges to 0 in the weak topology of $H^{1}$ and $\left(d_{I}^{*}(j)\right)_{j=1}^{\infty}$ converge to 0 in the $\mathrm{w}^{*}$ topology of $\left(H^{1}\right)^{*}$, there exists an index $j_{0}$ such that

$$
\operatorname{supp}\left(d_{I}^{\left(j_{0}\right)}\right)=X_{I}, \quad 2^{-j_{0}}<\min \left\{|K|: K \in \bigcup_{i=1}^{k} \mathcal{E}_{i}\right\},
$$

as well as

$$
\operatorname{dist}_{H^{1}}\left(d_{I}^{\left(j_{0}\right)}, W_{I}\right)<\eta_{I}, \quad \operatorname{dist}_{\left(H^{1}\right)^{*}}\left(d_{I}^{*\left(j_{0}\right)}, G_{I}\right)<\eta_{I}
$$

Player (II) concludes step 2 by choosing the collection

$$
\mathcal{E}_{I}=\left\{K \in \mathcal{D}_{j_{0}}: K \subset X_{I}\right\}
$$

the numbers

$$
\lambda_{K}^{(I)}=|K| /|I|, \quad K \in \mathcal{E}_{I}, \quad \text { and } \quad \mu_{K}^{(I)}=1, \quad K \in \mathcal{E}_{I},
$$

and defining

$$
x_{I}=d_{I}^{\left(j_{0}\right)} \quad \text { and } \quad x_{I}^{*}=d_{I}^{*\left(j_{0}\right)} .
$$

Clearly, since $\left|X_{I}\right|=\left|\mathcal{E}_{I}^{*}\right|=|I|$, we have $\sum_{K \in \mathcal{E}_{I}} \lambda_{K}^{(I)} \mu_{K}^{(I)}=1$. We conclude turn $k+1=\mathcal{O}(I)$ with player $(\mathrm{I})$ choosing $\left(\varepsilon_{K}^{(I)}\right)_{K \in \mathcal{E}_{I}}$ in $\{-1,1\}^{\mathcal{E}_{I}}$ in step 3 . 
We claim that this defines a winning strategy for player (II). In fact, $\left(x_{I}\right)_{I \in \mathcal{D}}$ and $\left(x_{I}\right)_{I \in \mathcal{D}}^{*}$ were constructed using the Gamlen-Gaudet construction, for which it was verified in [18. Theorem 0] that $\left(x_{I}\right)_{I}$ is equivalent to $\left(e_{I}\right)_{I} \in \mathcal{D}$ in $H^{1}$ and $\left(x_{I}^{*}\right)_{I}$ is equivalent to $\left(e_{I}^{*}\right)_{I} \in \mathcal{D}$ in $\left(H^{1}\right)^{*}$. Moreover, we note that in the text above we already verified $\operatorname{dist}_{H^{1}}\left(x_{I}, W_{I}\right)<\eta_{I}$ and $\operatorname{dist}_{\left(H^{1}\right)^{*}}\left(x_{I}^{*}, G_{I}\right)<\eta_{I}, I \in \mathcal{D}$.

5.3. The Haar system in $H^{p}\left(H^{q}\right), 1 \leq p, q<\infty$. In 13] it was shown that the two-parameter tensor product Haar system in $H^{p}\left(H^{q}\right)$ has the factorization property. We use the techniques introduced in [13, to show that, moreover, the two-parameter tensor product Haar system is strategically reproducible $H^{p}\left(H^{q}\right)$. Hence, by Theorem 3.12 we recover the main result in 13. Finally, we remark that by exploiting Theorem 7.6 (see Section 7 below) we obtain a simpler construction than the one used in 13 .

In the proof below, we will use following notation: The $H^{p}\left(H^{q}\right)$-normalized biparameter Haar system $\left(e_{I} \otimes f_{J}\right)_{I, J}$ is given by $e_{I} \otimes f_{J}=h_{I} \otimes h_{J} /\left(|I|^{1 / p}|J|^{1 / q}\right)$, and its bi-orthogonal functionals $\left(\left(e_{I} \otimes f_{J}\right)^{*}\right)_{I, J}$ are given by $\left(e_{I} \otimes f_{J}\right)^{*}=e_{I}^{*} \otimes f_{J}^{*}=$ $h_{I} \otimes h_{J} /\left(|I|^{1 / p^{\prime}}|J|^{1 / q^{\prime}}\right)$, where $1 / p+1 / p^{\prime}=1$ and $1 / q+1 / q^{\prime}=1$, with the usual convention that $1 / \infty=0$.

Theorem 5.3. The normalized bi-parameter Haar system is strategically reproducible in the mixed norm Hardy spaces $H^{p}\left(H^{q}\right), 1 \leq p, q \leq \infty$.

Proof. Define the subspaces $V_{1}, V_{2}$ of $H^{p}\left(H^{q}\right)$ by

$$
V_{1}=\overline{\operatorname{span}\left\{h_{I \times J}:|I|<|J|\right\}} H^{p}\left(H^{q}\right) \quad \text { and } \quad V_{2}=\overline{\operatorname{span}\left\{h_{I \times J}:|I| \geq|J|\right\}} H^{p}\left(H^{q}\right) \text {, }
$$

and note that $V_{1} \oplus V_{2}=H^{p}\left(H^{q}\right)$. We will now show that the bi-parameter Haar system is strategically reproducible in $V_{1}$ and in $V_{2}$, separately.

CASE $V_{1}$. Here, we will show that the bi-parameter Haar system is strategically reproducible in $V_{1}$. Player (I) opens the game by selecting $\eta_{[0,1 / 2) \times[0,1)}>0$, $W_{[0,1 / 2) \times[0,1)} \in \operatorname{cof}\left(H^{p}\left(H^{q}\right)\right)$ and $G_{[0,1 / 2) \times[0,1)} \in \operatorname{cof}_{w^{*}}\left(\left(H^{p}\left(H^{q}\right)\right)^{*}\right)$. In step 2, player (II) will select one of the following collections of dyadic rectangles $\mathcal{E}_{[0,1 / 2) \times[0,1)}^{(j)}$, $j \in \mathbb{N}$, given by

$$
\mathcal{E}_{[0,1 / 2) \times[0,1)}^{(j)}=\left\{K \times[0,1): K \in \mathcal{D}_{j}, K \subset[0,1 / 2)\right\}, \quad j \in \mathbb{N} .
$$

To this end, define

$$
\begin{aligned}
& d_{[0,1 / 2) \times[0,1)}^{(j)}=\sum_{K \times L \in \mathcal{E}_{[0,1 / 2) \times[0,1)}^{(j)}} \frac{|K|^{1 / p}|L|^{1 / q}}{|[0,1 / 2)|^{1 / p}|[0,1)|^{1 / q}} e_{K} \otimes f_{L}, \quad j \in \mathbb{N}, \\
& d_{[0,1 / 2) \times[0,1)}^{*(j)}=\sum_{K \times L \in \mathcal{E}_{[0,1 / 2) \times[0,1)}^{(j)}} \frac{|K|^{1 / p^{\prime}}|L|^{1 / q^{\prime}}}{|[0,1 / 2)|^{1 / p^{\prime}}|[0,1)|^{1 / q^{\prime}}} e_{K}^{*} \otimes f_{L}^{*}, \quad j \in \mathbb{N},
\end{aligned}
$$

and note that $K \times L \in \mathcal{E}_{[0,1 / 2) \times[0,1)}^{(j)}$ implies $L=[0,1)$. By Lemma 4.2 the sequence $\left(d_{[0,1 / 2) \times[0,1)}^{(j)}\right)_{j=1}^{\infty}$ is a null sequence in the weak topology of $H^{p}\left(H^{q}\right)$ and $\left(d_{[0,1 / 2) \times[0,1)}^{*(j)}\right)_{j=1}^{\infty}$ is a null sequence in the $\mathrm{w}^{*}$ topology of $H^{p}\left(H^{q}\right)^{*}$, we can find $j_{0}$, such that

$$
\operatorname{dist}_{H^{p}\left(H^{q}\right)}\left(d_{[0,1 / 2) \times[0,1)}^{\left(j_{0}\right)}, W_{[0,1 / 2) \times[0,1)}\right)<\eta_{[0,1 / 2) \times[0,1)},
$$




$$
\operatorname{dist}_{\left(H^{p}\left(H^{q}\right)\right)^{*}}\left(d_{[0,1 / 2) \times[0,1)}^{*\left(j_{0}\right)}, G_{[0,1 / 2) \times[0,1)}\right)<\eta_{[0,1 / 2) \times[0,1)} .
$$

Player II completes step 2 by selecting

$$
\mathcal{E}_{[0,1 / 2) \times[0,1)}=\mathcal{E}_{[0,1 / 2) \times[0,1)}^{\left(j_{0}\right)}
$$

and the numbers

$$
\begin{aligned}
\lambda_{K \times L}^{[0,1 / 2) \times[0,1)} & =\frac{|K|^{1 / p}|L|^{1 / q}}{|[0,1 / 2)|^{1 / p}|[0,1)|^{1 / q}}, & & K \times L \in \mathcal{E}_{[0,1 / 2) \times[0,1)}, \\
\mu_{K \times L}^{[0,1 / 2) \times[0,1)} & =\frac{|K|^{1 / p^{\prime}}|L|^{1 / q^{\prime}}}{|[0,1 / 2)|^{1 / p^{\prime}}|[0,1)|^{1 / q^{\prime}}}, & & K \times L \in \mathcal{E}_{[0,1 / 2) \times[0,1)} .
\end{aligned}
$$

Finally, in step 3, player (I) chooses

$$
\left(\varepsilon_{K \times L}^{[0,1 / 2) \times[0,1)}\right)_{K \times L \in \mathcal{E}_{[0,1 / 2) \times[0,1)}} \in\{-1,+1\}^{\mathcal{E}_{[0,1 / 2) \times[0,1)},}
$$

completing turn 1 .

Assume that the turns $1, \ldots, k$ of the game have been played out. We will now describe turn $k+1$. Select $I, J \in \mathcal{D}$ such that $|I|<|J|$ and $I \times J$ is the $(k+1)^{\text {st }}$ rectangle of the set $\{K \times L:|K|<|L|\}$ in the order $\triangleleft$. Player I starts off the turn by choosing $\eta_{I \times J}>0, W_{I \times J} \in \operatorname{cof}\left(H^{p}\left(H^{q}\right)\right)$ and $G_{I \times J} \in \operatorname{cof}_{w^{*}}\left(\left(H^{p}\left(H^{q}\right)\right)^{*}\right)$. In step 2, player (II) will select one of the collections $\mathcal{E}_{I \times J}^{(j)}, j \in \mathbb{N}$ of dyadic rectangles, which we will now describe in detail. We will distinguish between the following two cases: $J=[0,1)$ and $J \neq[0,1)$.

If $J=[0,1)$, we note that $I \neq[0,1)$, hence, $\mathcal{E}_{\widetilde{I} \times[0,1)}$ has already been defined. Also note that $[0,|I|) \times[0,1) \unlhd I \times[0,1)$, and choose the integer $\kappa(I \times[0,1))$ such that

$$
2^{-\kappa(I \times[0,1))}<\min \left\{|K|: K \times L \in \bigcup_{i=1}^{k} \mathcal{E}_{i}\right\} .
$$

For all $j \in \mathbb{N}$, we define

$$
\mathcal{E}_{I \times[0,1)}^{(j)}= \begin{cases}\left\{K^{+} \times[0,1): K \in \mathcal{D}_{j}, K \subset[0,1) \subset \mathcal{E}_{\widetilde{I} \times[0,1)}\right\}, & \text { if } I=(\widetilde{I})^{\ell} \\ \left\{K^{-} \times[0,1): K \in \mathcal{D}_{j}, K \subset[0,1) \subset \mathcal{E}_{\widetilde{I} \times[0,1)}\right\}, & \text { if } I=(\widetilde{I})^{r},\end{cases}
$$

where $J^{\ell}$ denotes the left successor and $J^{r}$ the right successor of a dyadic interval $J \in \mathcal{D}$. Define the functions

$$
\begin{aligned}
& d_{I \times[0,1)}^{(j)}=\sum_{K \times L \in \mathcal{E}_{I \times[0,1)}^{(j)}} \frac{|K|^{1 / p}|L|^{1 / q}}{|I|^{1 / p}|[0,1)|^{1 / q}} e_{K} \otimes f_{L}, \quad j \in \mathbb{N}, \\
& d_{I \times[0,1)}^{*(j)}=\sum_{K \times L \in \mathcal{E}_{I \times[0,1)}^{(j)}} \frac{|K|^{1 / p^{\prime}}|L|^{1 / q^{\prime}}}{|I|^{1 / p^{\prime}}|[0,1)|^{1 / q^{\prime}}} e_{K}^{*} \otimes f_{L}^{*}, \quad j \in \mathbb{N},
\end{aligned}
$$

and note that by Lemma $4.2\left(d_{I \times[0,1)}^{(j)}\right)_{j=1}^{\infty}$ converges to 0 in the weak topology of $H^{p}\left(H^{q}\right)$ and $\left(d_{I \times[0,1)}^{*(j)}\right)_{j=1}^{\infty}$ converges to 0 in the $\mathrm{w}^{*}$ topology of $\left(H^{p}\left(H^{q}\right)\right)^{*}$. Hence, there exists and index $j_{0}>\kappa(I \times[0,1))$ such that

$$
\begin{array}{r}
\operatorname{dist}_{H^{p}\left(H^{q}\right)}\left(d_{I \times[0,1)}^{\left(j_{0}\right)}, W_{I \times[0,1)}\right)<\eta_{I \times[0,1)}, \\
\operatorname{dist}_{\left(H^{p}\left(H^{q}\right)\right)^{*}}\left(d_{I \times[0,1)}^{*\left(j_{0}\right)}, G_{I \times[0,1)}\right)<\eta_{I \times[0,1)} .
\end{array}
$$


Player II then completes step 2 by putting

$$
\mathcal{E}_{I \times[0,1)}=\mathcal{E}_{I \times[0,1)}^{\left(j_{0}\right)}
$$

and selecting the numbers

$$
\begin{aligned}
& \lambda_{K \times L}^{I \times[0,1)}=|K|^{1 / p}|L|^{1 / q} /\left(|I|^{1 / p}|[0,1)|^{1 / q}\right), \\
& \mu_{K \times L}^{I \times[0,1)}=|K|^{1 / p^{\prime}}|L|^{1 / q^{\prime}} /\left(|I|^{1 / p^{\prime}}|[0,1)|^{1 / q^{\prime}}\right) .
\end{aligned}
$$

If $J \neq[0,1)$, then $\mathcal{E}_{I \times[0,1)}$ has already been defined. The collection $\mathcal{E}_{I \times J}$ will be chosen as one of the following collections $\mathcal{E}_{I \times J}^{(j)}, j \in \mathbb{N}$, which are given by

$$
\mathcal{E}_{I \times J}^{(j)}=\left\{K \times L: K \in \mathcal{D}_{j}, K \times L \subset \mathcal{E}_{I \times[0,1)}\right\}, \quad j \in \mathbb{N} .
$$

To this end, define the functions

$$
\begin{array}{ll}
d_{I \times J}^{(j)}= & \sum_{K \times L \in \mathcal{E}_{I \times J}^{(j)}} \frac{|K|^{1 / p}|L|^{1 / q}}{|I|^{1 / p}|J|^{1 / q}} e_{k} \otimes f_{L}, \quad j \in \mathbb{N}, \\
d_{I \times J}^{*(j)}= & \sum_{K \times L \in \mathcal{E}_{I \times J}^{(j)}} \frac{|K|^{1 / p^{\prime}}|L|^{1 / q^{\prime}}}{|I|^{1 / p^{\prime}}|J|^{1 / q^{\prime}}} e_{k}^{*} \otimes f_{L}^{*}, \quad j \in \mathbb{N},
\end{array}
$$

and note that by Lemma $4.2\left(d_{I \times J}^{(j)}\right)_{j=1}^{\infty}$ converges to 0 in the weak topology of $H^{p}\left(H^{q}\right)$ and that $\left(d_{I \times J}^{(j)}\right)_{j=1}^{\infty}$ converges to 0 in the $\mathrm{w}^{*}$ topology of $\left(H^{p}\left(H^{q}\right)\right)^{*}$. Consequently, we can find an index $j_{0}$ such that

$$
2^{-j_{0}}<\min \left\{|K|: K \times L \in \bigcup_{i=1}^{k} \mathcal{E}_{i}\right\},
$$

and

$$
\operatorname{dist}_{H^{p}\left(H^{q}\right)}\left(d_{I \times J}^{\left(j_{0}\right)}, W_{I \times J}\right)<\eta_{I \times J}, \text { and } \operatorname{dist}_{\left(H^{p}\left(H^{q}\right)\right)^{*}}\left(d_{I \times J}^{*\left(j_{0}\right)}, G_{I \times J}\right)<\eta_{I \times J} .
$$

Player II completes step 2 by selecting

$$
\mathcal{E}_{I \times J}=\mathcal{E}_{I \times J}^{\left(j_{0}\right)}
$$

and the numbers

$$
\lambda_{K \times L}^{I \times J}=\frac{|K|^{1 / p}|L|^{1 / q}}{|I|^{1 / p}|J|^{1 / q}} \quad \text { and } \quad \mu_{K \times L}^{I \times J}=\frac{|K|^{1 / p^{\prime}}|L|^{1 / q^{\prime}}}{|I|^{1 / p^{\prime}}|J|^{1 / q^{\prime}}},
$$

for all $K \times L \in \mathcal{E}_{I \times J}$.

Finally, in both cases $(J=[0,1)$ and $J \neq[0,1))$ player (I) completes step 3 (and thereby turn $(k+1))$ player (I) chooses

$$
\left(\varepsilon_{K \times L}^{I \times J}\right)_{K \times L \in \mathcal{E}_{I \times J}} \in\{-1,+1\}^{\mathcal{E}_{I \times J}} .
$$

CASE $V_{2}$. Follows from a completely parallel argument to Case $V_{1}$. 


\section{The HaAr SyStem in $L^{1}$ IS Strategically REPROdUCible}

In this section we consider the space $L^{1}$ of all absolutely integrable functions on $[0,1]$ instead of $L_{0}^{1}$. If we additionally define $h_{\emptyset}=\chi_{[0,1)}$ and $\mathcal{D}^{+}=\mathcal{D} \cup\{\emptyset\}$. We call then $\left(h_{I}\right)_{I \in \mathcal{D}^{+}}$is a monotone Schauder basis of $L^{1}$, if ordered lexicographically (i.e., $\emptyset$ is the minimum of $\mathcal{D}^{+}$and the rest of the order is inherited from the lexicographical order of $D$ ). The reason we consider $L^{1}$ is that we can prove an isometric statement in this setting and it is unclear whether this is possible for the space $L_{0}^{1}$. The purpose is to prove that the normalized Haar system of $L^{1}$ is strategically reproducible, and thus has the factorization property. The main difficulty is proving the following statement.

Theorem 6.1. The normalized Haar system of $L^{1}$ is 1-strategically reproducible.

The proof of the above will be presented at it in its own Subsection 6.2. We will also need the following statement.

Proposition 6.2. The normalized Haar system of $L^{1}$ has the $1 / \delta$-diagonal factorization property.

We will give the proof of Proposition 6.2 in Subsection 6.1. For the time being, we use the above two results to prove the following Corollary.

Corollary 6.3. The normalized Haar system of $L^{1}$ has the $1 / \delta$-factorization property.

Proof. By Theorem 6.1 the normalized Haar system is 1-strategically reproducible and by Proposition 6.2 it has the $1 / \delta$-factorization property. Since the Haar system is monotone, Theorem 3.12 yields that it has the $1 / \delta$-factorization property.

The following is a direct consequence of Corollary 6.3 .

Corollary 6.4. The normalized bi-parameter Haar system of $L^{1}\left(L^{1}\right)$ (which is isometrically isomorphic to $\left.L^{1}\left([0,1]^{2}\right)\right)$ has the $1 / \delta$-factorization property.

Proof. Let $T: L^{1}\left(L^{1}\right) \rightarrow L^{1}\left(L^{1}\right)$ be a bounded linear operator so that for all $I, J \in \mathcal{D}$ we have

$$
\left|\iint\left(h_{I} \otimes h_{J}\right) T\left(\frac{1}{|I||J|} h_{I} \otimes h_{J}\right) d x d y\right| \geq \frac{1}{\delta} .
$$

The space $L^{1}\left(L^{1}\right)$ is the projective tensor product of $L^{1}$ with itself. This means that if we consider any two bounded linear operators $R, S$ on $L^{1}$ then there is a (unique) bounded linear operator $R \otimes S: L^{1}\left(L^{1}\right) \rightarrow L^{1}\left(L^{1}\right)$ satisying $(R \otimes S)(f \otimes g)=$ $(R f) \otimes(S g)$ and $\|R \otimes S\|=\|R\|\|S\|$.

Consider the canonical projection $P_{[0,1)}$ from $L^{1}$ onto the linear span of $h_{\emptyset}$, which has norm one, and also consider the identity $I_{L^{1}}$ on $L^{1}$. Take the map $P=P_{[0,1)} \otimes I_{L^{1}}: L^{1}\left(L^{1}\right) \rightarrow L^{1}\left(L^{1}\right)$, which satisfies $\|P\|=1$. Its image is the space $Y=\left[h_{\emptyset} \otimes h_{I}\right]_{I \in \mathcal{D}}$, which is naturally isometric to $L^{1}$ via the map $h_{\emptyset} \otimes h_{I} \mapsto h_{I}$. It follows that the map $P \circ T: Y \rightarrow Y$ may be identified with a map on $L^{1}$ with diagonal bounded below by $\delta$. This means, by Theorem 6.2 that for $\varepsilon>0$ there are $B: Y \rightarrow L^{1}, A: L^{1} \rightarrow Y$ so that $B(P T) A=I_{L^{1}}$ and $\|A\|\|B\| \leq(1+\varepsilon) / \delta$. Now, since $L^{1}$ and $L^{1}\left(L^{1}\right)$ are isometrically isomorphic we may take an onto isometry $Q: L^{1} \rightarrow L^{1}\left(L^{1}\right)$ and set $\tilde{A}=A Q^{-1}: L^{1}\left(L^{1}\right) \rightarrow L^{1}\left(L^{1}\right), \tilde{B}=Q B P: L^{1}\left(L^{1}\right) \rightarrow$ $L^{1}\left(L^{1}\right)$. It follows that $\|\tilde{A}\|\|\tilde{B}\| \leq(1+\varepsilon) / \delta$ and $\tilde{B} T \tilde{A}=I$. 
Remark 6.5. One can use [8, Theorem 4.2] to give a, relatively, short proof of the following. There is $C \geq 1$ so that if $T: L^{1} \rightarrow L^{1}$ is a bounded linear operator with diagonal bounded below by $\delta$ then there are $A, B: L^{1} \rightarrow L^{1}$ with $B T A=I$ and $\|B\|\|A\| \leq C\|T\| / \delta^{2}$. There are two differences with Theorem 6.2. The first one is the power appearing on $\delta$. The more noticeable one is that in Theorem 6.2 the factorization does not depend on $\|T\|$. This seems to be the case for all known spaces with the factorization property.

Remark 6.6. We point out that Theorem 6.1, Proposition 6.2 and Corollary 6.3 are true for the normalized Haar system of $L^{p}[0,1], 1 \leq p<\infty$ as well. The proof of Theorem 6.1 requires only minor modifications and in certain cases it is simpler due to reflexivity and unconditionality. The proof of Proposition 6.2 is different and one has to use the details of the proof of Theorem 6.1. The factorization property of these spaces has been known since [1], however existing proofs did not give a sharp factorization estimate, in particular it was not known whether the Haar system of $L^{p}[0,1], 1 \leq p<\infty$, has the $C / \delta$-factorization property for a uniform constant $C \geq 1$.

6.1. The proof of Proposition 6.2, We now turn to the proof of Proposition 6.2. We divide the argument into several steps formulated in Lemma 6.7 and 6.9, below. Lemma 6.7 is likely to be know. We present a short proof for sake of completeness and convenience of the reader.

Lemma 6.7. Let $I_{0} \in \mathcal{D}$ and $Y_{I_{0}}=\left[\left(h_{I}\right)_{I \subset I_{0}}\right]$. Then there are a subspace $Z$ of $Y_{I_{0}}$ that isometrically isomorphic to $L^{1}$ and a norm one linear projection $P: L^{1} \rightarrow Z$.

Proof. Set $m_{0}=\inf I_{0}, M_{0}=\sup I_{0}$ and let $Z$ be the subspace consisting of all absolutely integrable functions $f$ that have support in $I_{0}$ and satisfy the condition

$$
f(x)=-f\left(\frac{m_{0}+M_{0}}{2}+x\right) \text { a.e. in } I_{0}^{+} .
$$

It follows that $W$ is a subspace of $Y_{I_{0}}$. An onto isometry $T: Z \rightarrow L^{1}$ is given by

$$
T f(x)=\left|I_{0}\right| f\left(m_{0}+\frac{\left|I_{0}\right|}{2} x\right) .
$$

The desired projection $P: L^{1} \rightarrow Z$ is defined as follows. For every $f \in L^{1}$ let $f_{1}=$ $\left.f\right|_{I_{0}^{+}}, f_{2}=\left.f\right|_{I_{0}^{-}}$, let $g_{1}$ be the function with support $I_{0}^{+}$so that $g_{1}(x)=f\left(x+\left|I_{0}\right| / 2\right)$, for $x \in I_{0}^{+}$, and let $g_{2}$ be the function with support $I_{0}^{-}$so that $g_{2}(x)=f\left(x-\left|I_{0}\right| / 2\right)$, for $x \in I_{0}^{-}$. One can check that $\left\|f_{1}+f_{2}\right\|_{L^{1}}=\left\|g_{1}+g_{2}\right\|_{L^{1}}=\|f\|_{L^{1}}$. It is also not hard to see that $\left(f_{1}+f_{2}\right)-\left(g_{1}+g_{2}\right)=\left(f_{1}-g_{1}\right)+\left(f_{2}-g_{2}\right)$ is in $W$. The final step is to observe that $P f=(1 / 2)\left[\left(f_{1}+f_{2}\right)-\left(g_{1}+g_{2}\right)\right]$ is a norm one projection onto $Z$.

It is common to call diagonal operators on the Haar system Haar multipliers. Loosely following 25 we use the following notation.

\section{Notation 6.8.}

(i) A chain of $\mathcal{D}^{+}$is a sequence of intervals $\mathcal{C}=\left(I_{n}\right)_{n}$ so that $I_{1} \supsetneq I_{2} \supsetneq \cdots$.

(ii) Given a Haar multiplier $D$ with entries $\left(c_{I}\right)_{I \in \mathcal{D}^{+}}$we define the quantity $\|D\|_{W}=\sup \sum_{n=1}^{\infty}\left|c_{I_{n}}-c_{I_{n+1}}\right|$, where the supremum is taken over all chains $\mathcal{C}=\left(I_{n}\right)_{n}$.

(iii) Given a Haar multiplier $D$ with entries $\left(c_{I}\right)_{I \in \mathcal{D}^{+}}$we define the quantity $\|D\|_{\infty}=\sup _{I \in \mathcal{D}^{+}}\left|c_{I}\right|$. 
Lemma 6.9. Let $D: L^{1} \rightarrow L^{1}$ be a bounded Haar multiplier with entries $\left(c_{I}\right)_{I \in \mathcal{D} \text {. }}$ Then for every $\varepsilon>0$ there exists $I_{0} \in \mathcal{D}$ so that if $Y_{I_{0}}=\left[\left(h_{I}\right)_{I \subset I_{0}}\right]$ then

$$
\left\|\left.\left(D-c_{I_{0}} I\right)\right|_{Y_{0}}\right\| \leq \varepsilon
$$

Proof. According to 25. Theorem 3] (see end of page 312), for any Haar multiplier $D: L^{1} \rightarrow L^{1}$ we have

$$
\frac{1}{4}\|D\|_{W} \leq\|D\| \leq\|D\|_{W}+3\|D\|_{\infty} .
$$

We use the above to choose $I_{0} \in \mathcal{D}$ with the property that for any chain $\mathcal{C}=\left(I_{n}\right)$ with $I_{n} \subset I_{0}$ we have $\sum_{n}\left|c_{I_{n}}-c_{I_{n+1}}\right| \leq \varepsilon / 4$. If such an $I_{0}$ would not exist then it would easily follow that $\|D\|_{W}=\infty$ which by (14) contradicts the boundedness of $D$.

Let $P_{I_{0}}$ define the canonical projection onto $Y_{I_{0}}$, given by $P\left(\sum_{I \in \mathcal{D}} a_{I} h_{I}\right)=$ $\sum_{I \subset I_{0}} a_{I} h_{I}$, which has norm at most two. Clearly, $P_{I_{0}}$ is a Haar multiplier. Next, define the Haar multiplier $S$ with entries $\left(\tilde{c}_{I}\right)_{I \in \mathcal{D}^{+}}$, whith $\tilde{c}_{I}=c_{I}$ is $I \subset I_{0}$ and $\tilde{c}_{I}=0$ otherwise. It is easy to see that $\left\|S-c_{I_{0}} P_{I_{0}}\right\|_{W}=\|S\|_{W} \leq \varepsilon / 4$ and that $\left\|S-c_{I_{0}} P_{I_{0}}\right\|_{\infty} \leq \varepsilon / 4$. Therefore by (14) we deduce $\left\|S-P_{I_{0}}\right\| \leq \varepsilon$. Since $\left.D\right|_{Y_{I_{0}}}=\left.S\right|_{Y_{I_{0}}}$ and and $\left.I\right|_{Y_{I_{0}}}=\left.P_{I_{0}}\right|_{Y_{I_{0}}}$ we finally conclude $\left\|\left.\left(D-c_{I_{0}} I\right)\right|_{Y_{I_{0}}}\right\|=$ $\left\|\left.\left(S-c_{I_{0}} P_{I_{0}}\right)\right|_{Y_{I_{0}}}\right\| \leq\left\|S-c_{I_{0}} P_{I_{0}}\right\| \leq \varepsilon$.

We are ready to conclude this subsection with a proof of Proposition 6.2

Proof of Proposition 6.2. Let $D$ be a bounded Haar multiplier on $L^{1}$ satisfying $\inf _{I \in \mathcal{D}^{+}}\left|c_{I}\right| \geq \delta>0$ and fix $\varepsilon>0$. We will show that the identity $(1+\varepsilon) / \delta$-factors through $D$. Use Lemma 6.9 to find $I_{0} \in \mathcal{D}$ so that $\left\|\left.\left(D-c_{I_{0}} I\right)\right|_{Y_{I_{0}}}\right\| \leq \delta \varepsilon /(1+\varepsilon)$.

By Lemma 6.7 there are a subspace $Z$ of $Y_{I_{0}}$, an onto isometry $A: L^{1} \rightarrow Z$, and a norm one projection $P: L^{1} \rightarrow Z$. Define $B=\left(1 / c_{I_{0}}\right) A^{-1} P$, which is well defined on $L^{1}$ and $\|B\|=\left|1 / c_{I_{0}}\right| \leq 1 / \delta$. As the image of $A$ is $Z$ it easily follows that $B\left(c_{I_{0}} I\right) A=I$. We calculate

$$
\|B D A-I\|=\left\|B\left(D-c_{I_{0}} I\right) A\right\|=\left\|\left.B\left(D-c_{I_{0}} I\right)\right|_{Z} A\right\| \leq\|B\|\|A\| \frac{\delta \varepsilon}{1+\varepsilon} \leq \frac{\varepsilon}{1+\varepsilon}
$$

and hence the operator $B D A$ is invertible with $\left\|(B D A)^{-1}\right\| \leq 1+\varepsilon$. Define $\tilde{B}=$ $(B D A)^{-1} B$. Observe that $\tilde{B} D A=I$ and $\|\tilde{B}\|\|A\| \leq(1+\varepsilon) / \delta$, i.e., the identity almost $1 / \delta$-factors through $D$.

6.2. The proof of Theorem 6.1. The following Lemma 6.10 is a well known result, which goes back to Gamlen-Gaudet [10]. For more details, we also refer to [21, page $176 \mathrm{ff}$.]. It describes the situation player (II) is striving to achieve in order to win the game of strategic reproducibility. Recall that for $\mathcal{A} \subset \mathcal{D}$ we set $\mathcal{A}^{*}=\cup \mathcal{A}$.

Lemma 6.10. Let $\kappa \in(0,1)$. Then, there exists a sequence of positive real numbers $\left(\delta_{n}\right)_{n}$ so that the following holds. Let $\left(\mathcal{H}_{I}\right)_{I \in \mathcal{D}^{+}}$be a collection of non-empty finite subsets of $\mathcal{D}$, so that for each $I \in \mathcal{D}^{+}$the collection $\mathcal{H}_{I}$ consists of pairwise disjoint intervals, and for each $I \in \mathcal{D}^{+}$let $\bar{\varepsilon}_{I}=\left(\varepsilon_{L}^{I}\right)_{L \in \mathcal{H}_{I}} \in\{-1,1\}^{\mathcal{H}_{I}}$. Define for each $I \in \mathcal{D}$ the function $b_{I}=\sum_{L \in \mathcal{H}_{I}} \varepsilon_{L}^{I} h_{L}$ and $b_{\emptyset}=\left|\sum_{L \in \mathcal{H}_{\emptyset}} \varepsilon_{L}^{I} h_{L}\right|$. Assume that the following are satisfied.

(a) For all $I, J \in \mathcal{D}$ with $I \cap J=\emptyset$ we have $\mathcal{H}_{I}^{*} \cap \mathcal{H}_{J}^{*}=\emptyset$.

(b) For all $I \in \mathcal{D}$ we have $\operatorname{supp}\left(b_{I^{+}}\right) \subset\left[b_{I}=1\right]$ and $\operatorname{supp}\left(b_{I^{-}}\right) \subset\left[b_{I}=-1\right]$. 
(c) For all $n \in \mathbb{N}$ and $I \in \mathcal{D}_{n}$ if $I=J^{+}$or $I=J^{-}$we have

$$
\left(1-\delta_{n}\right) \frac{\left|\mathcal{H}_{J}^{*}\right|}{2} \leq\left|\mathcal{H}_{I}^{*}\right| \leq\left(1+\delta_{n}\right) \frac{\left|\mathcal{H}_{J}^{*}\right|}{2} .
$$

(d) $\mathcal{H}_{[0,1)}^{*} \subset \mathcal{H}_{\emptyset}^{*}$ and $\left|\mathcal{H}_{[0,1)}^{*}\right| \geq\left(1-\delta_{1}\right)\left|\mathcal{H}_{\emptyset}^{*}\right|$.

Then, if $\lambda=\left|\mathcal{H}_{\emptyset}^{*}\right|$, the sequences $\left(h_{I} /|I|\right)_{I \in \mathcal{D}^{+}}$and $\left(b_{I} / \lambda|I|\right)_{I \in \mathcal{D}^{+}}$, when they are both viewed as sequences in $L^{1}$, are $(1+\kappa)$-impartially equivalent. Furthermore, the sequences $\left(b_{I}\right)_{I \in \mathcal{D}^{+}}$and $\left(h_{I}\right)_{I \in \mathcal{D}^{+}}$, when they are both viewed as a sequence in $L^{\infty}$, are isometrically equivalent.

The following Lemma allows player (II) to make the appropriate choice of vectors.

Lemma 6.11. Let $H$ be in $\operatorname{cof}\left(L^{1}\right), G$ be in $\operatorname{cof}_{w^{*}}\left(L^{\infty}\right)$, and $\kappa>0$. Then there exists $n_{0} \in \mathbb{N}$ so that for every $f$ in the linear span of $\left(h_{I}\right)_{I \in \mathcal{D} \backslash \mathcal{D}^{n_{0}}}$ with $\|f\|_{\infty} \leq 1$ we have

(i) $\operatorname{dist}(f, H)<\kappa$, if $f$ is viewed as an element of $L^{1}$ and

(ii) $\operatorname{dist}(f, H)<\kappa$, if $f$ is viewed as an element of $L^{\infty}$.

Proof. We first show (i). Recall that there are $g_{1}, \ldots, g_{N} \in L^{\infty}$ so that $H=$ $\cap_{j=1}^{N} \operatorname{ker} g_{j}$. It follows, from the Hahn Banach theorem, that there is $\delta>0$ so that for every $f \in L^{1}$ with $\left|\int g_{j}(x) f(x) d x\right|<\delta$ for $1 \leq j \leq N$ we have $\operatorname{dist}(f, H)<\kappa$. If we assume that the conclusion is false, i.e. the desired $k_{0}$ does not exist, there is a sequnce $\left(f_{k}\right)_{k}$ with $\left\|f_{k}\right\|_{\infty} \leq 1$ and $f_{k} \in \operatorname{span}\left\{h_{I}: I \in \mathcal{D} \backslash \mathcal{D}^{k}\right\}$, so that $\operatorname{dist}\left(f_{k}, H\right) \geq \kappa$ for all $k \in \mathbb{N}$. As this sequence is uniformly integrable it has a subsequence $\left(f_{k_{i}}\right)_{i}$ that converges weakly to an $f \in \cap_{k} \overline{\operatorname{span}}\left\{h_{I}: I \in \mathcal{D} \backslash \mathcal{D}^{k}\right\}=\{0\}$. Thus, $\lim _{i} \mid \int g_{j}(x) f_{k_{i}}(x) d x=0$, for $1 \leq j \leq N$, i.e. $\lim _{k} \operatorname{dist}\left(f_{k_{i}}, H\right)=0$, which is a contradiction.

The second statement follows from a similar argument. Use that there are $g_{1}, \ldots, g_{N}$ in $L^{1}$ so that $G=\left\{f_{1}, \ldots, f_{N}\right\}^{\perp}$ and that for any sequence $\left(g_{k}\right)_{k}$ with $\left\|g_{k}\right\|_{\infty} \leq 1$ and $g_{k} \in \operatorname{span}\left\{h_{I}: I \in \mathcal{D} \backslash \mathcal{D}^{k}\right\}$, for all $k \in \mathbb{N}$, we have that $\left(g_{k}\right)_{k}$ converges to zero in the $w^{*}$-topology.

We refer to Section 4.5 for the notation employed systematically in the proof, below.

Proof of Theorem 6.1. Enumerate $\mathcal{D}^{+}$as $\left(I_{k}\right)_{k \in \mathbb{N}}$ according to lexicographical order. We will describe the winning strategy of player (II) in a game of $\operatorname{Rep}_{\left.\left(L_{0}^{1}, h_{I}\right)\right)}(1, \eta)$, for fixed $\eta>0$. Before the game starts player (I) picks a partition $\mathbb{N}=N_{1} \cup N_{2}$, which corresponds to a partition $\mathcal{D}^{+}=\mathcal{A}_{1} \cup \mathcal{A}_{2}$. Before proceeding with the game, we claim that $[0,1)=\limsup \left(A_{1}\right) \cup \limsup \left(A_{2}\right)$. Indeed, if $x \in[0,1)$ then for every $I \in \mathcal{D}$ with $x \in I$ we have $I \in \mathcal{A}_{1}$ or $I \in \mathcal{A}_{2}$. That is, $x \in I$ for infinitely many $I \in \mathcal{A}_{1}$ or for infinitely many $I \in \mathcal{A}_{2}$. In the first case $x \in \lim \sup \left(A_{1}\right)$ and in the second case $x \in \lim \sup \left(A_{2}\right)$. We proceed by assuming without loss of generality that $\left|\lim \sup \left(\mathcal{A}_{1}\right)\right| \geq 1 / 2$. By Lemma 4.4 there is $\mathcal{A} \subset \mathcal{A}_{1}$ so that $\mathscr{G}_{n}(\mathcal{A})$ is finite for all $n \in \mathbb{N}$ and $|\lim \sup (\mathcal{A})|=\lambda \geq 2 / 3$. Henceforth, when we use Notation 4.3 it shall be with respect to the collection $\mathcal{A}$. This collection $\mathcal{A}$ corresponds to some $N \subset N_{1}$. Each round $k$ corresponds to an $I \in \mathcal{D}^{+}$via the lexicographical identification $\mathcal{D}^{+} \leftrightarrow \mathbb{N}$. Player (I) first chooses $\eta_{k}>0, W_{k} \in \operatorname{cof}\left(L^{1}\right)$ and $G_{k} \in \operatorname{cof}_{w^{*}}\left(L^{\infty}\right)$. Then player (II) has the right to choose a subset $E_{k}$ of either $N_{1}$ or $N_{2}$. He or she will always choose $E_{k} \subset N$. This $E_{k}$ corresponds to a finite $\mathcal{H}_{I} \subset \mathcal{A}$. We shall describe the choice in detail further bellow, but let us say for the time being that 
there is $m_{k} \in \mathbb{N}$, with $m_{k}>m_{k-1}$ if $k>1$, so that $\mathcal{H}_{I} \subset \mathcal{A}_{m_{k}}$ and $\mathcal{H}_{I}^{*} \subset \mathcal{H}_{\emptyset}^{*}$. Next, player (I) chooses signs $\left(\varepsilon_{i}^{k}\right)_{i \in E_{k}}$ which we relabel as $\left(\varepsilon_{L}^{I}\right)_{L \in \mathcal{H}_{I}}$. We shall put $b_{I}=\sum_{L \in \mathcal{H}_{I}} \varepsilon_{L}^{I} h_{I}$ and $\tilde{b}_{I}=b_{\emptyset} b_{I}$ (pointwise). If $k>1$ then by the fact that $m_{k}>1$ and $\mathcal{H}_{I}^{*} \subset \mathcal{H}_{\emptyset}^{*}$ we have that $\tilde{b}_{I}=\sum_{L \in \mathcal{H}_{I}} \tilde{\varepsilon}_{L}^{I} h_{I}$, for a choice of signs $\left(\tilde{\varepsilon}_{L}^{I}\right)_{I \in \mathcal{H}_{I}}$ that does not necessarily coincide with $\left(\varepsilon_{L}^{I}\right)_{L \in \mathcal{H}_{I}}$. Of some importance is also the sequence of positive real numbers $\left(\delta_{n}\right)_{n}$ provided by Lemma 6.10 if we take $\kappa=\eta$.

We can now describe how player (II) makes a choice in each round $k$. Let $I \in \mathcal{D}^{+}$ correspond to $k$ in the lexicographical enumeration. Then, either $I=\emptyset$ (if $k=1$ ), $I=[0,1)$ (if $k=2$ ), or there is $2 \leq k^{\prime}<k$ so that if $J=I_{k^{\prime}}$ then $I=J^{+}$or $I=J^{-}$. The round starts by player (I) picking $\eta_{k}>0$. Player (II) will pick $E_{k} \subset N$ that corresponds to an $\mathcal{H}_{I} \subset \mathcal{A}$ which is chosen as follows:

(i) There is $m_{k} \in \mathbb{N}$, with $m_{k}>m_{k-1}$ if $k>1$, so that $\mathcal{H}_{I}$ is of one of the following forms

(ia) $\mathcal{H}_{I}=\mathscr{G}_{m_{k}}(\mathcal{A})$, if $I=\emptyset$ or $I=[0,1)$ (i.e., when $k=1$ or $k=2$ ),

(ib) $\mathcal{H}_{I}=\left(\mathcal{H}_{J}\right)_{\bar{\varepsilon}_{J}, m_{k}}^{\text {succ }}$, if $I=J^{+}=I_{k^{\prime}}$ and $\bar{\varepsilon}_{J}=\left(\tilde{\varepsilon}_{L}^{J}\right)_{J \in \mathcal{H}_{J}}$ coming from $\tilde{b}_{J}$.

(ic) $\mathcal{H}_{I}=\left(\mathcal{H}_{J}\right)_{-\bar{\varepsilon}_{J}, m_{k}}^{\text {succ }}$, if $I=J^{-}=I_{k^{\prime}}$ and $\bar{\varepsilon}_{J}=\left(\tilde{\varepsilon}_{L}^{J}\right)_{J \in \mathcal{H}_{J}}$ coming from $\tilde{b}_{J}$.

(ii) If $A=\lim \sup (\mathcal{A})$ then if $\left|\mathcal{H}_{I}^{*} \cap A\right|>\left(1-\delta_{n+1} / 2\right)\left|\mathcal{H}_{I}^{*}\right|$, where $I \in \mathcal{D}_{n}$ and $\left(\delta_{i}\right)_{i}$ is the sequence mentioned above, provided by Lemma 6.10. If $I=\emptyset$ replace $n$ with 0 .

(iii) For every $f \in \operatorname{span}\left(h_{L}\right)_{L \in \mathcal{D} \backslash \mathcal{D}^{m_{k}-1}}$ with $\|f\|_{\infty} \leq 1$ we have

$$
\operatorname{dist}_{L^{1}}\left(f, W_{k}\right) \leq \eta_{k}|I| \text { and } \operatorname{dist}_{L^{\infty}}\left(f, G_{k}\right) \leq \eta_{k} .
$$

Having chosen such an $\mathcal{H}_{I}$ player (II) picks scalars $\left(\lambda_{L}^{I}\right)_{L \in \mathcal{H}_{I}},\left(\mu_{L}^{I}\right)_{L \in \mathcal{H}_{I}}$ by taking $\lambda_{L}^{I}=|L| /\left(\left|\mathcal{H}_{\emptyset}\right||I|\right)$ and $\mu_{L}^{I}=1$, for all $L \in \mathcal{H}_{I}$.

We must show that player (II) can pick $\mathcal{H}_{I}$ satisfying (i), (ii), and (iii) as well as that

$$
1-\eta \leq \sum_{L \in \mathcal{H}_{I}} \lambda_{L}^{I} \mu_{L}^{I} \leq 1+\eta
$$

Note that if $m_{k}$ is sufficiently large then by Lemma 6.11 condition (iii) is satisfied. We can focus on showing that we can pick $m_{k}$, as large as desired, so that $\mathcal{H}_{I}$ is of one of the forms in (i) and so that (ii) is satisfied. If $I=\emptyset$ and $k=1$ then $A=\cap_{m} \mathscr{G}_{m}(\mathcal{A})^{*}$ then $|A|=\lim _{n}\left|\mathscr{G}_{m}(\mathcal{A})^{*}\right|$ which easily yields that we can pick $m_{1}$ as large as we wish so that $\left|\mathscr{G}_{m_{1}}(\mathcal{A})^{*} \cap A\right|=|A|>\left(1-\delta_{2} / 2\right)\left|\mathscr{G}_{m_{1}}(\mathcal{A})^{*}\right|$. If $I=[0,1)$ we act similarly. If $I=J^{+}$with $I \in \mathcal{D}_{n}$ then by assumption player (II) has picked $\mathcal{H}_{J} \subset \mathcal{A}$ with $\left|\mathcal{H}_{J}^{*} \cap A\right|>\left(1-\delta_{n} / 2\right)\left|\mathcal{H}_{J}^{*}\right|$. Let also $\bar{\varepsilon}_{J}=\left(\tilde{\varepsilon}_{L}^{J}\right)_{J \in \mathcal{H}_{J}}$ denote the signs coming from $\tilde{b}_{J}$. By Lemma 4.5 there exists $m_{0}$ so that for any $m \geq m_{0}$ we have

$$
\left|\left(\left(\mathcal{H}_{J}\right)_{\bar{\varepsilon}_{J}, m}^{\text {succ }}\right)^{*} \cap A\right|>\left(1-\delta_{n+1} / 2\right)\left|\left(\left(\mathcal{H}_{J}\right)_{\bar{\varepsilon}_{J}, m}^{\text {succ }}\right)^{*}\right| .
$$

Thus, if we pick $m_{k}$ sufficiently large we may set $\mathcal{H}_{I}=\left(\mathcal{H}_{J}\right)_{\bar{\varepsilon}_{J}, m}^{\text {succ }}$ and (i) and (ii) are satisfied. If $I=J^{-}$the argument is the same.

The proof of 15 requires an inductive argument. We will show this simultaneously with proving that player (II) has forced the desired winning conditions. Define for $I \in \mathcal{D}^{+}$the functions

$$
x_{I}=\sum_{L \in \mathcal{H}_{I}} \varepsilon_{L}^{I} \frac{|L|}{\left|\mathcal{H}_{\emptyset}\right||I|} h_{I} \in L^{1} \text { and } x_{I}^{*}=\sum_{L \in \mathcal{H}_{I}} \varepsilon_{L}^{I} h_{I} \in L^{\infty} .
$$


Out next goal is to show that $\left(x_{I}\right)_{I \in \mathcal{D}^{+}}$is $(1+\eta)$-impartially equivalent to $\left(h_{I} /|I|\right)_{I \in \mathcal{D}^{+}}$ in $L^{1}$ and $\left(x_{I}^{*}\right)_{I \in \mathcal{D}}$ is isometrically equivalent to $\left(h_{I}\right)_{I \in \mathcal{D}}$ in $L^{\infty}$. We will first show that $\left(\tilde{b}_{I}\right)_{I \in \mathcal{D}^{+}}$, where $\tilde{b}_{\emptyset}=b_{\emptyset}^{2}=\left|\sum_{L \in \mathcal{H}_{\emptyset}} \varepsilon_{L}^{\emptyset} h_{L}\right|$ and for each $I \in \mathcal{D} \tilde{b}_{I}=$ $\sum_{L \in \mathcal{H}_{I}} \tilde{\varepsilon}_{L}^{I} h_{I}$, satisfies the assumptions of Lemma 6.10 and use that to reach the desired goal.

Assumption (a) follows easily from (i) and assumption (b) follows easily from (ib) and (ic). Let us now show that (c) is satisfied and let $n \in \mathbb{N}, I \in \mathcal{D}_{n}$, with $I=I_{k}$, so that $I=J^{+}$or $I=J^{-}$for some $J \in \mathcal{D}_{n-1}$. We shall assume that $I=J^{+}$as the other case has the same proof. By (iii), applied to $J$, we have that $\left|\mathcal{H}_{J}^{*} \cap A\right|>\left(1-\delta_{n} / 2\right)\left|\mathcal{H}_{J}^{*}\right|$. This, by the first statement of Lemma 4.5 yields that

$$
\left|\left(\mathcal{H}_{J}\right)_{\bar{\varepsilon}_{J}}^{*} \cap A\right|>\left(1-\delta_{n}\right) \frac{\left|\mathcal{H}_{J}^{*}\right|}{2} .
$$

By the definition of $\left(\mathcal{H}_{J}\right)_{\bar{\varepsilon}_{J}, m_{k}}^{\text {succ }}$ it follows that $\left(\mathcal{H}_{J}\right)_{\bar{\varepsilon}_{J}}^{*} \cap A=\left(\mathcal{H}_{J}\right)_{\bar{\varepsilon}_{J}, m_{k}}^{*} \cap A=\mathcal{H}_{I}^{*} \cap A$. We calculate,

$$
\frac{\left|\mathcal{H}_{J}^{*}\right|}{2}=\left|\left(\mathcal{H}_{J}\right)_{\bar{\varepsilon}_{J}}^{*}\right| \geq\left|\mathcal{H}_{I}^{*}\right| \geq\left|\mathcal{H}_{I}^{*} \cap A\right|>\left(1-\delta_{n}\right) \frac{\left|\mathcal{H}_{J}^{*}\right|}{2},
$$

i.e., (c) holds. Assumption (d) is easier to show.

Now that we know that the assumptions of Lemma 6.10 are satisfied we conclude that the sequences

$$
\tilde{x}_{I}=\sum_{L \in \mathcal{H}_{I}} \tilde{\varepsilon}_{L}^{I} \frac{|L|}{\left|\mathcal{H}_{\emptyset}\right||I|} h_{I}=\in L^{1} \text { and } \tilde{x}_{I}^{*}=\sum_{L \in \mathcal{H}_{I}} \tilde{\varepsilon}_{L}^{I} h_{I} \in L^{\infty} .
$$

are $(1+\eta)$-impartially equivalent to $\left(h_{I} /|I|\right)_{I \in \mathcal{D}^{+}}$in $L^{1}$ and isometrically equivalent to $\left(h_{I}\right)_{I \in \mathcal{D}}$ in $L^{\infty}$ respectivelly. We next observe that for $I \in \mathcal{D}^{+}$we have $\tilde{x}_{I}=b_{\emptyset} x_{I}$ and $\tilde{x}_{I}^{*}=b_{\emptyset} x_{I}^{*}$. But $\left|b_{\emptyset}(t)\right|$ is one whenever $t \in \mathcal{H}_{\emptyset}^{*} \supset \operatorname{supp}\left(x_{I}\right)=\operatorname{supp}\left(x_{I}^{*}\right)$ and thus $\left(x_{I}\right)_{I \in \mathcal{D}^{+}}$is isometrically equivalent to $\left(\tilde{x}_{I}\right)_{I \in \mathcal{D}^{+}}$and $\left(x_{I}^{*}\right)_{I \in \mathcal{D}^{+}}$is isometrically equivalent to $\left(\tilde{x}_{I}^{*}\right)_{I \in \mathcal{D}^{+}}$. This means that we reached our goal.

Next, we need to observe that if $I=I_{k}$ then $\operatorname{dist}_{L^{1}}\left(x_{I}, W_{k}\right)<\eta_{k}$ as well as $\operatorname{dist}_{L^{\infty}}\left(x_{I}^{*}, G_{k}\right)<\eta_{k}$. Both of these inequalities are an immediate consequence of (iii) and the fact that $x_{k}, x_{k}^{*} \in \operatorname{span}\left(h_{L}\right)_{L \in \mathscr{G}_{n_{k}}(\mathcal{A})} \subset \operatorname{span}\left(h_{L}\right)_{L \in \mathcal{D} \backslash \mathcal{D}^{m_{k}-1}}$ and $\left\|x_{I}\right\|_{L^{\infty}}=|I|,\left\|x_{I}^{*}\right\|_{L^{\infty}}=1$.

It only remains to prove (15), which follows easily from the fact that $\left(x_{I}\right)_{I}$ is $(1+\eta)$-impartially equivalent to $\left(h_{I} /|I|\right)_{I}$. Indeed,

$$
\sum_{L \in \mathcal{H}_{I}} \lambda_{L}^{I} \mu_{L}^{I}=\frac{1}{\lambda|I|} \sum_{L \in \mathcal{H}_{I}}|L|=\left\|x_{I}\right\|_{L^{1}}
$$

and $\left\|x_{I}\right\|_{L^{1}}$ is between

$$
\sqrt{(1-\eta)}\left(\left\|h_{I}\right\|_{L^{1}} /|I|\right)>1-\eta \quad \text { and } \quad \sqrt{(1+\eta)}\left(\left\|h_{I}\right\|_{L^{1}} /|I|\right)<1+\eta .
$$

The proof is complete.

\section{UNCONDITIONAL SUMS OF SPACES WITH STRATEGICAL REPRODUCIBLE BASES}

In this section we determine that the strategical reproducibility is inherited by unconditional sums. 
For a Banach spaces $X$ with a 1-unconditional basis $\left(e_{n}\right)_{n}$ and a sequence of Banach spaces $\left(Y_{n}\right)_{n}$ we denote by $Z=\left(\sum Y_{n}\right)_{X}$ the Banach space of all sequences $z=\left(y_{n}\right)_{n}$ with $y_{n} \in Y_{n}$ for all $n \in \mathbb{N}$ and the quantity

$$
\|z\|=\left\|\sum_{n=1}^{\infty}\right\| y_{n}\left\|_{Y_{n}} e_{n}\right\|_{X}
$$

is well defined. For each $k \in \mathbb{N}$ let $P_{k}: Z \rightarrow Y_{k}$ denote the map given by $P_{k}\left(y_{n}\right)_{n}=$ $y_{k}$. The space $Y_{k}$ can be naturally isometrically identified with a subspace of $\mathrm{Z}$, namely the one consisting of all sequences which have all coordinates, except the $k$ 'th one, equal to zero. Thus, with this identification, $P_{k}$ is a norm one projection.

Remark 7.1. If $A_{n}: Y_{n} \rightarrow Y_{n}, n \in \mathbb{N}$, are bounded linear operators and $\sup _{n}\left\|A_{n}\right\|=$ $C<\infty$ then by 1 -unconditionality the map $A: Z \rightarrow Z$ with $A(z)=\sum_{n} A_{n} P_{n}(z)$ is bounded with $\|A\|=C$.

Remark 7.2. If there exists a common $\lambda \geq 1$ such that each $Y_{n}$ has a Schauder basis $\left(e_{i}^{(n)}\right)_{i}$ whose basis constant is bounded by $\lambda$ then there is an enumeration $\left(\tilde{e}_{i}\right)$ of $\left(\left(e_{i}^{(n)}\right)_{i}\right)_{n}$ that is Schauder basic whose basis constant at most $\lambda$. In fact, this is satisfied by any enumeration $\left(\tilde{e}_{i}\right)$ with the property that whenever $i<j$ if for some $n \in \mathbb{N}$ we have $e_{i}^{(n)}=\tilde{e}_{k_{i}^{(n)}}, e_{j}^{(n)}=\tilde{e}_{k_{j}^{(n)}}$ then $k_{i}^{(n)}<k_{j}^{(n)}$.

Lemma 7.3. Let $X$ be a Banach space with a 1-unconditional basis $\left(e_{n}\right)_{n},\left(Y_{n}\right)_{n}$ be a sequence of Banach spaces, and $Z=\left(\sum Y_{n}\right)_{X}$. Assume that there are common $\lambda \geq 1$ and $K:(0,+\infty) \rightarrow \mathbb{R}$ so that each $Y_{n}$ has a Schauder basis $\left(e_{i}^{(n)}\right)_{i}$ whose constant is at most $\lambda$ that has the $K(\delta)$-diagonal factorization property. Then the sequence $\left(\left(e_{i}^{(n)}\right)_{i}\right)_{n}$ is a Schauder basis (using the linear order defined in Remark 7.2) whose basis constant is at most $\lambda$ and it has the $K(\delta)$-diagonal factorization property.

Proof. Let $D: X \rightarrow X$ be a diagonal operator with respect to $\left(\left(e_{i}^{(n)}\right)_{i}\right)_{n}$ so that $\inf _{i, n}\left|e_{i}^{(n) *} D\left(e_{i}^{(n)}\right)\right|>\delta$. If follows that for each $n \in \mathbb{N}$ the map $D$ restricted on $Y_{n}$ is a diagonal operator $D_{n}$ so that $\inf _{i, n}\left|e_{i}^{(n) *}\left(D_{n}\left(e_{i}^{(n)}\right)\right)\right|>\delta$. For $\kappa>0$, by assumption, there exist $B_{n}, A_{n}: Y_{n} \rightarrow Y_{n}$ with $\left\|A_{n}\right\|\left\|B_{n}\right\| \leq K(\delta)+\kappa$ and $B_{n} D_{n} A_{n}$ is the identity map on $Y_{n}$. By scaling, we may assume that $\max \left\{\left\|A_{n}\right\|,\left\|B_{n}\right\|\right\} \leq$ $\sqrt{K(\delta)+\kappa}$ and hence the maps $A, B: Z \rightarrow Z$, with $A(z)=\sum_{n} A_{n} P_{n}(z)$ and $B(z)=\sum_{n} B_{n} P_{n}(z)$ are well defined with $\|A\|\|B\| \leq K(\delta)+\kappa$. It is easily verified that $I=B D A$.

Lemma 7.4. Let $X$ be a Banach space with a 1-unconditional basis $\left(e_{n}\right)_{n},\left(Y_{n}\right)_{n}$ be a sequence of Banach spaces, and $Z=\left(\sum Y_{n}\right)_{X}$. Fix $n \in \mathbb{N}$ and let $A$, $B$ be finite subsets of $Z$ and $Z^{*}$ respectively. Define

$$
\begin{aligned}
A_{n} & =\left\{P_{n}(x): x \in A\right\}, B_{n}=\left\{P_{n}^{*}\left(x^{*}\right): x^{*} \in B\right\}, \\
G & =A^{\perp}, H=\bigcap_{x^{*} \in B} \operatorname{ker}\left(x^{*}\right), G_{n}=A_{n}^{\perp}, W_{n}=\bigcap_{x^{*} \in B_{n}} \operatorname{ker}\left(x^{*}\right) .
\end{aligned}
$$

Then, for every $x \in Y_{n}$ and $x^{*} \in Y_{n}^{*}$ we have $\operatorname{dist}(x, H) \leq \operatorname{dist}\left(x, W_{n}\right)$ and $\operatorname{dist}\left(x^{*}, G\right) \leq \operatorname{dist}\left(x^{*}, G_{n}\right)$.

Proof. For every $y \in W_{n}$ it follows that $P_{n}(y) \in H$. Hence $\|x-y\| \geq \| P_{n}(x-$ $y)\|=\| x-P_{n}(y) \| \geq \operatorname{dist}(x, H)$ and so $\operatorname{dist}\left(x, W_{n}\right) \geq \operatorname{dist}(x, H)$. Similarly, for 
$f \in G_{n}$ we have $P_{n}^{*} f \in G$ and we conclude in the same manner that $\operatorname{dist}\left(x^{*}, G\right) \leq$ $\operatorname{dist}\left(x^{*}, G_{n}\right)$.

Proposition 7.5. Let $X$ be a Banach space with a 1-unconditional basis $\left(e_{n}\right)_{n}$, $\left(Y_{n}\right)_{n}$ be a sequence of Banach spaces, and $Z=\left(\sum Y_{n}\right)_{X}$. Assume that there are common $\lambda \geq 1$ and $C \geq 1$ so that each $Y_{n}$ has a $C$-strategically reproducible Schauder basis $\left(e_{i}^{(n)}\right)_{i}$ whose basis constant is at most $\lambda$. Then the sequence $\left(\left(e_{i}^{(n)}\right)_{i}\right)_{n}$ enumerated as $\left(\tilde{e}_{n}\right)$ according to Remark 7.2 is a $C$-strategically reproducible Schauder basis whose basis constant is at most $\lambda$.

Proof. For each $n \in \mathbb{N}$ let $M_{n}$ be the infinite subset of $\mathbb{N}$ so that $\left(\tilde{e}_{i}\right)_{i \in M_{n}}=$ $\left(e_{i}^{(n)}\right)_{i}$. Here, we will now describe the winning strategy of player (II) in a game $\operatorname{Rep}_{\left(X,\left(\tilde{e}_{i}\right)\right)}(C, \eta)$. Let player (I) pick a partition $\mathbb{N}=N_{1} \cup N_{2}$. Note that for each $n \in \mathbb{N} M_{n}=M_{n}^{1} \cup M_{n}^{2}$, where $M_{n}^{1}=N_{1} \cap M_{n}, M_{n}^{2}=N_{2} \cap M_{n}$. The $m$ 'th round is played out as follows. Player (I) selects $\eta_{m}>0$ as well as $W_{n} \in \operatorname{cof}(Z)$, $G_{n} \in \operatorname{cof}_{w^{*}}\left(Z^{*}\right)$. Then, there are finite subsets $A_{m}$ and $B_{m}$ of $Z$ and $Z^{*}$ respectively so that $G_{m}=A_{m}^{\perp}$ and $W_{m}=\left(B_{m}\right)_{\perp}$. If $m \in M_{n}$, for some $n \in \mathbb{N}$, and $m$ is the $k^{\prime}$ th element of $M_{n}$ then set $A_{k}^{(n)}=\left\{P_{n} x: x \in A\right\}$ and $B_{k}^{(n)}=\left\{P_{n}^{*} f: f \in B\right\}$. Then set $G_{k}^{(n)}=\left(A_{k}^{(n)}\right)^{\perp}, W_{k}^{(n)}=\left(B_{k}^{(n)}\right)^{\perp}$. Let player (II) treat this round as the $k$ 'th round of a game $\operatorname{Rep}_{\left(Y_{n},\left(e_{i}^{(n)}\right)\right)}(C, \eta)$ and follow a winning strategy. In the end, for each $n \in \mathbb{N}$, player (II) has chosen $\left(x_{k}^{(n)}\right)_{k}$ in $Y_{n}$ and $\left(x_{k}^{(n) *}\right)_{k}$ in $Y_{n}^{*}$ so that

(i) the sequences $\left(x_{k}^{(n)}\right)_{k}$ and $\left(e_{k}^{(n)}\right)_{k}$ are impartially $(C+\eta)$-equivalent,

(ii) the sequences $\left(x_{k}^{(n) *}\right)_{k}$ and $\left(e_{k}^{(n) *}\right)_{k}$ are impartially $(C+\eta)$-equivalent,

(iii) for all $k \in \mathbb{N}$, if the $k^{\prime}$ th element of $M_{n}$ is $m$, we have $\operatorname{dist}\left(x_{k}^{(n)}, W_{k}^{n}\right)<\eta_{m}$,

(iv) for all $k \in \mathbb{N}$, if the $k^{\prime}$ th element of $M_{n}$ is $m$, we have $\operatorname{dist}\left(x_{k}^{(n) *}, G_{k}^{n}\right)<\eta_{m}$.

If we relabel $\left(x_{k}^{(n)}\right)_{k}$ as $\left(\tilde{x}_{m}\right)_{m \in M_{n}}$ and stitch them all together to a sequence $\left(\tilde{x}_{m}\right)_{m \in \mathbb{N}}$ then it easily follows that this sequence is impartially $(C+\eta)$-equivalent to $\left(\tilde{e}_{m}\right)_{m}$. Also by Lemma 7.4 we have $\operatorname{dist}\left(x_{m}, W_{m}\right)<\eta_{m}$, for all $m \in \mathbb{N}$. Similarly, relabel $\left(x_{k}^{(n) *}\right)_{k}$ as $\left(\tilde{x}_{m}^{*}\right)_{m \in M_{n}}$ and take $\left(\tilde{x}_{m}^{*}\right)_{m}$, which is $(C+\eta)$-impartially equivalent to $\left(\tilde{e}_{m}^{*}\right)_{m}$. Also, $\operatorname{dist}\left(x_{m}^{*}, G_{m}\right)<\eta_{m}$, for all $m \in \mathbb{N}$. In other words, player two has emerged victorious.

Theorem 7.6. Let $X$ be a Banach space with a 1-unconditional basis $\left(e_{n}\right)_{n},\left(Y_{n}\right)_{n}$ be a sequence of Banach spaces, and $Z=\left(\sum Y_{n}\right)_{X}$. Assume that there are common $\lambda \geq 1, C \geq 1$, and $K:(0,+\infty) \rightarrow \mathbb{R}$ so that each $Y_{n}$ has a Schauder basis $\left(e_{i}^{(n)}\right)_{i}$ that satisfies the following:

(i) its basis constant is at most $\lambda$,

(ii) it has the $K(\delta)$-diagonal factorization property and

(iii) it is $C$-strategically reproducible in $Y_{n}$.

Then the sequence $\left(\left(e_{i}^{(n)}\right)_{i}\right)_{n}$ enumerated as $\left(\tilde{e}_{n}\right)$ (using the linear order defined in Remark 7.2) has the $\lambda C^{2} K(\delta)$-factorization property.

Proof. By Lemma 7.3 the basis of $Z$ is $\lambda$-basic and it has the $K(\delta)$-diagonal factorization property. By Proposition 7.5 the basis of $Z$ is $C$-strategically reproducible. We finish off the proof by using Proposition 7.5 .

Remark 7.7. A consequence of the above theorem is that if one takes $X=\ell_{p}$, $1 \leq p<\infty$ of $X=c_{0}$ and a sequence of spaces $\left(Y_{n}\right)_{n}$, with each $Y_{n}$ being some 
$L^{p}$ or $\ell_{p}, 1 \leq p<\infty$, or $c_{0}$ then $Z=\left(\sum Y_{n}\right)_{X}$ has a 1-strategically reproducible basis. Of course, $X$ can be any space of an unconditional basis and this produces an interesting example. It was proved in [13] that Gowers' space $X$ with an unconditional basis from [11 does not satisfy the factorization property, however, for this $X$ and any sequence $\left(Y_{n}\right)_{n}$ as before the space $Z=\left(\sum Y_{n}\right)_{X}$ does satisfy it.

\section{FinAl COMMENTS AND OPEN PROBLEMS}

Capon [4] showed that the bi-parameter Haar system in $L^{p}\left(L^{q}\right), 1<p, q<$ $\infty$, has the factorization property. With refined techniques, Capon's result was extended to $H^{1}\left(H^{1}\right)$ by 20 and then later in 13 to $H^{p}\left(H^{1}\right)$ and $H^{1}\left(H^{p}\right), 1<p<$ $\infty$. In Section 5 , we gave a different proof of their results in, by writing $H^{p}\left(H^{q}\right)$ as a complemented sum of two spaces, solving the problem in each of the components separately, and then using the fact that strategically reproducibility is inherited by complemented sums. This begs the following question.

Problem 8.1. If $X$ and $Y$ are Banach spaces with bases that have the factorization property, does the union of those two bases (in the right order) have the factorization property in the complemented sum of $X$ and $Y$ ?

As we remarked after Theorem 3.12 the uniform factorization property from Definition 3.10 is formally stronger than the factorization property from Definition 2.3 (iii).

Problem 8.2. Is there a Banach space with a basis that satisfies the factorization property and fails the uniform factorization property?

In Corollary 6.4 we showed that the bi-parameter Haar system has the factorization property. Nevertheless, we do not know the answer to the following problem.

Problem 8.3. Is the normalized bi-parameter Haar system of $L^{1}\left(L^{1}\right)$ strategically reproducible?

The unit vector basis of Tsirelson space $T$, i.e. the space constructed by Figiel and Johnson in 9], which is the dual of Tsirelson's original space, is not strategically reproducible. This follows from the following two facts. On the one hand, every block bases $\left(x_{i}\right)$ is equivalent to a subsequence $\left(e_{n_{i}}\right)$ with $n_{i} \in \operatorname{supp}\left(x_{i}\right)$, $i \in \mathbb{N}$. Secondly, if the subsequence is an Ackerman sequence (i.e. is increasing fast enough), then $\left[e_{n_{i}}\right]$ is not isomorphic to $T$. Thus, if player (I) chooses an Ackerman sequence in the game described in Definition 3.3. he wins. This leads to the next problem.

Problem 8.4. Does the unit vector basis in $T$ have the factorization property?

Among all the bi-parametric Lebesgue and Hardy spaces, $L^{p}\left(L^{1}\right)$ and $L^{1}\left(L^{p}\right)$, $1<p<\infty$, seem to resist our approaches.

Problem 8.5. Is the bi-parameter Haar system strategically reproducible or does it at least have the factorization property in $L^{p}\left(L^{1}\right)$ and $L^{1}\left(L^{p}\right), 1<p<\infty$ ?

More generally, if $X$ has a basis which is strategically reproducible, or has the factorization property, does the tensor product of that basis with the Haar system in $L^{p}(X), 1 \leq p<\infty$ have the same property?

In this context it is worth noting that Capon [5] proved $L^{p}(X), 1 \leq p<\infty$ is primary, if $X$ has a symmetric basis. 


\section{REFERENCES}

[1] A. D. Andrew, Perturbations of Schauder bases in the spaces $C(K)$ and $L^{p}, p>1$, Studia Math. 65 (1979), no. 3, 287-298.

[2] D. Alspach, P. Enflo, and E. Odell, On the structure of separable $\mathcal{L}_{p}$ spaces $(1<p<\infty)$, Studia Math. 60 (1977), no. 1, 79-90.

[3] S. Argyros, P. Motakis, and B. Sari, A study of conditional spreading sequences, J. Funct. Anal. 273 (2017), no. 3, 1205-1257, DOI 10.1016/j.jfa.2017.04.009.

[4] M. Capon, Primarité de $L^{p}\left(L^{r}\right), 1<p, r<\infty$, Israel J. Math. 42 (1982), no. 1-2, 87-98.

[5] _ Primarité de $L^{p}(X)$, Trans. Amer. Math. Soc. 276 (1983), no. 2, 431-487, DOI $10.2307 / 1999061$.

[6] P. G. Casazza, W. B. Johnson, and L. Tzafriri, On Tsirelson's space, Israel J. Math. 47 (1984), 81-98.

[7] P. G. Casazza and T. J. Shura, Tsirel'son's space, Lecture Notes in Mathematics, vol. 1363, Springer-Verlag, Berlin, 1989. With an appendix by J. Baker, O. Slotterbeck and R. Aron.

[8] P. Enflo and T. W. Starbird, Subspaces of $L^{1}$ containing $L^{1}$, Studia Math. 65 (1979), 203225.

[9] T. Figiel and W. B. Johnson, A uniformly convex Banach space which contains no $l_{p}$, Compositio Math. 29 (1974), 179-190.

[10] J. L. B. Gamlen and R. J. Gaudet, On subsequences of the Haar system in $L_{p}[1,1](1 \leq p \leq$ $\infty)$, Israel J. Math. 15 (1973), 404-413.

[11] W. T. Gowers, A solution to Banach's hyperplane problem, Bull. London Math. Soc. 26 (1994), no. 6, 523-530, DOI 10.1112/blms/26.6.523.

[12] J.-P. Kahane, Some random series of functions, Second, Cambridge Studies in Advanced Mathematics, vol. 5, Cambridge University Press, Cambridge, 1985.

[13] N. J. Laustsen, R. Lechner, and P. F. X. Müller, Factorization of the identity through operators with large diagonal, J. Funct. Anal. (2018), https://doi.org/10.1016/j.jfa.2018.02.010.

[14] J. Marcinkiewic, Quelques theorèmes sur les séries orthogonales, Ann. Soc. Polon. Math. 16 (1937), 8596.

[15] D. A. Martin, Borel determinacy, Ann. of Math. (2) 102 (1975), no. 2, 363-371, DOI $10.2307 / 1971035$.

[16] B. Maurey, Système de Haar, Séminaire Maurey-Schwartz 1974-1975: Espaces Lsup p, applications radonifiantes et géométrie des espaces de Banach, Exp. Nos. I et II, 1975, pp. 26 pp. (erratum, p. 1).

[17] _ Sous-espaces complémentés de $L^{p}$, d'après P. Enflo, Séminaire Maurey-Schwartz 1974-1975: Espaces $L^{p}$, applications radonifiantes et géométrie des espaces de Banach, Exp. No. III, 1975 , pp. 15 pp. (erratum, p. 1).

[18] P. F. X. Müller, On subsequences of the Haar basis in $H^{1}(\delta)$ and isomorphism between $H^{1}$-spaces, Studia Math. 85 (1986), no. 1, 73-90.

[19] - On projections in $H^{1}$ and BMO, Studia Math. 89 (1988), no. 2, 145-158.

[20] 38 (1994), no. 4, 554-573.

[21] Isomorphisms between $H^{1}$ spaces, Instytut Matematyczny Polskiej Akademii Nauk. Monografie Matematyczne (New Series) [Mathematics Institute of the Polish Academy of Sciences. Mathematical Monographs (New Series)], vol. 66, Birkhäuser Verlag, Basel, 2005.

[22] E. Odell and Th. Schlumprecht, Trees and branches in Banach spaces, Trans. Amer. Math. Soc. 354 (2002), 4085-4108 (electronic).

[23] R.E.A.C. Paley, A remarkable series of orthogonal functions, Proc. London Math, Soc. 34 (1932), 241-264.

[24] A. Pełczyński, Projections in certain Banach spaces, Studia Math. 19 (1960), 209-228.

[25] E. M. Semenov and S. N. Uksusov, Multipliers of series in the Haar system, Sibirsk. Mat. Zh. 53 (2012), no. 2, 388-395. 
R. Lechner, Institute of Analysis, Johannes Kepler University Linz, Altenberger Strasse 69, A-4040 Linz, Austria

E-mail address: Richard.Lechner@jku.at

P. Motakis, Department of Mathematics, University of Illinois at Urbana-Champaign, URBANA, IL 61801, U.S.A.

E-mail address: pmotakis@illinois.edu

P.F.X. Müller, Institute of Analysis, Johannes Kepler University Linz, Altenberger Strasse 69, A-4040 Linz, Austria

E-mail address: Paul.Mueller@jku.at

Th. Schlumprecht, Department of Mathematics, Texas A\&M University, College Station, TX 77843-3368, USA, and Faculty of Electrical Engineering, Czech Technical University in Prague, Zikova 4, 16627, Prague, Czech Republic

E-mail address: schlump@math.tamu.edu 